\title{
A study of composite laminates failure using an anisotropic gradient-enhanced damage mean-field homogenization model
}

\author{
L. Wu ${ }^{\mathrm{a}, *}$, F. Sket ${ }^{\mathrm{d}}$, J.M. Molina-Aldareguia ${ }^{\mathrm{d}}$, A. Makradi ${ }^{\mathrm{e}}$, L. Adam ${ }^{\mathrm{b}}$, I. Doghri ${ }^{\mathrm{b}, \mathrm{c}}$, L. Noels $^{\mathrm{a}}$ \\ ${ }^{a}$ University of Liege, Department of Mechanical and Aerospace Engineering, Computational 83 Multiscale Mechanics of Materials, Chemin \\ des Chevreuils 1, B-4000 Liège, Belgium \\ ${ }^{b}$ e-Xstream Engineering, Axis Park-Building H, Rue Emile Francqui 9, B-1435 Mont-Saint-Guibert, Belgium \\ ${ }^{c}$ Université catholique de Louvain, Institute of Mechanics, Materials and Civil Engineering (iMMC), Avenue G. Lemaître 4, 1348 \\ Louvain-la-Neuve, Belgium \\ ${ }^{d}$ IMDEA Materials Institute, C/ Eric Kandel 2, 28906 Getafe, Spain \\ ${ }^{e}$ Centre de Recherche Public Henri Tudor, Avenue John F. Kennedy, 29, L-1855 Luxembourg, Luxembourg
}

\begin{abstract}
The failure of carbon fiber reinforced epoxy laminates is studied using an anisotropic gradient-enhanced continuum damage model embedded in a mean-field homogenization scheme.

In each ply, a homogenized material law is used to capture the intra-laminar failure. The anisotropy of the homogenized material model results from the homogenization method and from the reformulation of the non-local continuum damage theory to account for the material anisotropy. As a result the damage propagation direction in each ply is predicted with accuracy as compared to the experimental results, while the problems of losing uniqueness and strain localization, which occur in classical finite element simulations when strain softening of materials is involved, can be avoided.

To model the delamination process, the hybrid discontinuous Galerkin/extrinsic cohesive law method is introduced at the ply interfaces. This hybrid method avoids the need to propagate topological changes in the mesh with the propagation of the delamination while it preserves the consistency and stability in the un-cracked interfaces.

As a demonstration, open-hole coupons with different stacking sequences are studied numerically and experimentally. Both the intra- and inter-laminar failure patterns are shown to be well captured by the computational framework.
\end{abstract}

Keywords: Damage, Composite laminates, Mean-Field Homogenization, Multi-scale, Delamination

\section{Introduction}

An efficient design using advanced composite materials relies on the development of accurate analytical and computational tools which are able to predict the response of composite structures under complex severe loading conditions. In particular, the modeling of the failure process of such structures becomes an important requirement to reduce the cost inherent to mechanical tests on large numbers of specimens.

Fracture mechanisms of composite materials are complex and require a multiscale approach: from the microscale within a ply to the laminate macroscale. Different solutions have been developed to address these particular topics, such as the damage-based micro-meso-macro approaches for composites [1-3], or purely numerical approaches as discussed by LLorca et al. [4]. However, since

\footnotetext{
${ }^{*}$ Corresponding author, Phone: +32 436694 53, Fax: +324366 9505

Email addresses: L.Wu@ulg.ac.be (L. Wu ),

Federico.sket@imdea.org (F. Sket), jon.molina@imdea.org (J.M. Molina-Aldareguia), ahmed.makradi@tudor.lu (A. Makradi),

laurent.adam@e-xstream.com (L. Adam),

issam.doghri@uclouvain.be (I. Doghri), L.Noels@ulg.ac.be (L. Noels)
}

composite materials are aggregates of multiple phases, and as each phase has its own mechanical properties, the failure models require the material characterization of many parameters at the laminate level $[2,3,5,6]$. Moreover, the use of mesh-dependent characteristic sizes $[5,6]$ or of time regularization [7] is common to simulate the failure of composite structures since the governing partial differential equations lose ellipticity at damage induced strainsoftening onset, removing the uniqueness of the finite element solution, which becomes mesh-dependent. As a result, the finite-element solution does not converge with the mesh refinement. The use of cohesive zones within the plies using the extended finite element method, see e.g. [8], or the phantom node method [9] does not suffer from the loss of solution uniqueness.

An alternative to these approaches consists in using homogenized material properties at the macro-scale, in which case only the constituents need to be characterized. Indeed the macro or mesoscopic material responses of heterogeneous materials can be derived from the micro-structure constituents properties using analytical and/or numerical homogenization techniques. A comprehensive overview of different homogenization methods can be found in References $[10,11]$. 
One semi-analytical efficient homogenization framework for the modeling of particle or fiber reinforced composite materials is the mean-field homogenization (MFH) approach [12]. MFH methods were first developed for linear elastic structures by extending the Eshelby single inclusion solution [13] to multiple inclusions interacting in an average way in the composite material. Most common extensions of the Eshelby solution are the Mori-Tanaka scheme $[14,15]$ and the self-consistent scheme [16, 17]. As the constituents of a composite material can exhibit an inelastic behavior such as plasticity, visco-elasticity, etc., MFH methods were also developed in the non-linear range [18-26].

Although multiscale homogenization methods in general, and $\mathrm{MFH}$ schemes in particular, have achieved a high level of accuracy to capture the non-linear behavior of composite materials, accounting for material degradation, through damage or fracture models, remains highly challenging, see the reviews in $[4,11]$. Recently, $\mathrm{Wu}$ et al. $[27,28]$ have proposed a non-local MFH accounting for the damage evolution of the matrix phase of the composite material. In that formulation, an incremental-secant $\mathrm{MFH}$ approach was developed in order to account for the elastic unloading of one of the composite material phases during the strain softening of the other phase. When compared to a finite-element resolution of the micro-structure, the model was shown to predict accurately the softening response of the composite material, as well as the response of the different phases, even for volume ratios of inclusions around $60 \%,[28]$. In order to avoid the strain/damage localization caused by the matrix material softening, an implicit non-local formulation [29-32] was adopted during the homogenization process.

Beside the intra-laminar damage, the inter-laminar failure is also of importance. The delamination process is usually modeled by recourse to cohesive interface elements inserted between the plies, which integrate an intrinsic cohesive law (ICL), see [33-38] among many others. In that case, the traction separation law of the cohesive element also models the elastic response prior to the delamination process, yielding mesh-dependent effects [37, 38] due to the lack of consistency of the method (because of the lack of consistency, convergence is not achieved upon mesh-refinement). This mesh-dependent effect has motivated the use of extrinsic cohesive laws (ECL), which represent the fracturing response only, and for which cohesive elements are inserted at the fracture onset $[39,40]$, requiring on-the-fly topological changes of the mesh. An energetically rigorous and computationally efficient way to integrate a cohesive zone model is to combine the extrinsic cohesive law with a discontinuous Galerkin (DG) approach [41-45]. With this hybrid method, interface elements are inserted between bulk elements at the beginning of the simulation, but the consistency and continuity during the pre-fracture stage are ensured by having recourse to the discontinuous interface terms, contrarily to a classical intrinsic cohesive zone model, thus avoiding the mesh-dependence effect and the need to propagate topological changes in the mesh with the propagation of the delamination. Efficient implementations of this method in open-source and commercial software are now available [46-48].

In this paper, the non-local damage-enhanced $\mathrm{MFH}$ model developed in $[27,28]$ is applied to predict the failure of composite laminates such as coupon tests with a hole. To this end numerical models of the laminates are obtained by meshing each different ply separately. Within a ply, the material model follows the MFH, allowing to represent the damage process corresponding to the different fiber orientations. At the interface between plies, the hybrid discontinuous Galerkin/extrinsic cohesive law (DG/ECL) method is used to model the delamination process. This paper is organized in two main sections.

Section 2 presents the multi-scale numerical model of the composite laminate. First, in Section 2.1, the implicit non-local approach originally derived for isotropic materials $[29-32]$ is extended to the case of anisotropic materials, such as UD-fiber reinforced epoxy ones. Indeed, in that case a single characteristic length $l$ is not enough to characterize the interactions, of the non-local model, between material points in all the directions. In the transverse directions of UD-fiber reinforced epoxy composites, the fibers have the effect of blocking the material points interactions, and on the contrary, in the longitudinal direction, fibers prolong the interactions between material points $[49,50]$. In order to respect this anisotropic character of composite materials, the implicit non-local approach is derived in an anisotropic framework, and three characteristic lengths $l^{1}, l^{2}$ and $l^{3}$ are defined in the three principal directions of the materials. The anisotropy of the homogenized material model results thus from the homogenization method and from the reformulation of the nonlocal continuum damage theory to account for the material anisotropy. Because of this anisotropic model, the damage propagates along the fiber directions, which is not necessarily the case with meso-scale continuum damage models [51]. As a result the damage propagation direction in each ply is predicted with accuracy as compared to the experimental results. Then, in Section 2.2, the finite-element discretization of the hybrid discontinuous Galerkin/extrinsic cohesive law method is presented in the particular case of laminate structures: within a ply a classical continuous Galerkin approximation is used while the hybrid DG/ECL method is used at ply interfaces, allowing the delamination to be captured by an adequate cohesive law. Third, the non-local incremental-secant MFH scheme, accounting for the damage evolution in the matrix [28], is recalled in Section 2.3 to define the material model of the bulk elements within the plies. Finally, as the delamination models are usually developed for intrinsic cohesive laws [33-38], their extrinsic version is proposed in Section 2.4.

Section 3 presents the numerical applications of the developed framework and its validation. The material parameters of the carbon fibers and of the epoxy matrix of 
prepreg Hexply M10.1/38\% / UD300 / HS (R) are identified from the manufacturer data and the cohesive energy required for the delamination model is extracted from a Double Cantilever Beam (DCB) experiment in Section 3.1. The effect of the characteristic lengths of the non-local damage model is studied in Section 3.2, showing the necessity to consider an anisotropic non-local model for UD composite material. Eventually the predicted responses of open-hole laminate coupons are compared to experimental results. Tensile tests on open-hole laminate involve complex intra- and inter-laminar failure modes which depend on the geometrical dimensions, stacking sequence etc. For this reason such tests have been commonly used to validate composite laminate failure models, as in [52] to assess the meso-scale continuum damage model [1], in [53] to validate the phantom-node method [53] combined to interface elements and fiber damage models, or in [54] to study the thickness size effect using the smeared crack model [5], as a non-exhaustive list. In the present work, experimental tests with two different stacking sequences are performed following the setup described in Section 3.3, and the results are compared to the developed model predictions in Section 3.4. It is found that the numerical model predicts the damage bands in locations and with orientations as observed in the experimental samples loaded up to $90 \%$ of the failure stress and inspected by X-ray computed tomography (XCT). Moreover, the fracture load is relatively well captured by the model as well as the delamination pattern.

\section{Multiscale model for composite laminates}

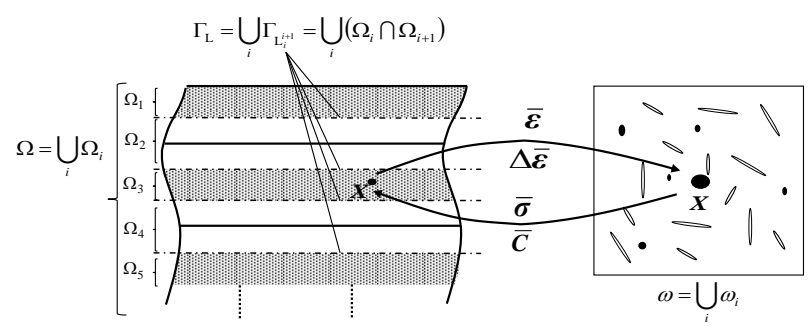

Figure 1: Multiscale method.

The laminate $\Omega$ is made of different plies $\Omega_{i}$ which are separated by interfaces $\Gamma_{L_{i}^{i+1}}$, see Fig. 1 . The unions of the interfaces between the plies is referred to as $\Gamma_{\mathrm{L}}$. At the macro-scale, each ply can be seen as an anisotropic homogeneous continuum assuming the separation of scales holds. For a complex material behavior, the material response within a ply can be obtained through a multiscale approach as illustrated in Fig. 1. At each macro-point $\boldsymbol{X}$ of the structure, the macro-strain tensor $\bar{\varepsilon}$ is known, and the macro-stress tensor $\overline{\boldsymbol{\sigma}}$ is sought from the resolution of a micro-scale boundary value problem (BVP). In this paper the macroscopic fields obtained from the subsequent homogenization process are over-lined whereas the microscopic fields are not. At the micro-level, the macro-point is viewed as the center of a representative volume element (RVE) of domain $\omega$ made of different phases $\omega_{i}$.

In the present paper the multiscale laminate problem is solved as follows. At the macro-scale the homogenized material law of each ply follows a damage-enhanced elastoplastic anisotropic model. In order to capture the softening response, this model is formulated in a non-local implicit form [29-31]. This non-local form is herein reformulated using anisotropic matrix length scales in order to account for the anisotropic behavior of the plies. At the microscale, the incremental-secant mean-field homogenization formulation developed for composite materials whose components obey to the elasto-plastic damage governing law is considered $[26,28]$. As delamination can occur between the plies, along $\Gamma_{L}$, the weak form of the non-local macroscale governing equations is formulated using a DG/ECL framework allowing the traction separation delamination law to be integrated.

\subsection{Anisotropic non-local gradient model}

To avoid the loss of objective solutions during strain softening, many enhanced physical or phenomenological models were proposed. As an example, the smeared formulation uses the characteristic length of the finite elements in the constitutive model [55]. Other continuum damage modeling approaches, avoiding the introduction of a mesh-dependent parameter, are supplied with a higherorder formulation, such as in the Cosserat model [56], the non-local model [57] or the gradient model [58].

Besides these damage formulations avoiding the loss of the solution uniqueness, the so-called implicit non-local approach was pioneered in [29-31]. In this formulation, some internal variables $a$ (which can be strains, accumulated plastic strain, damage...) are replaced by their weighted average $\tilde{a}$ over a characteristic volume $\left(V_{C}\right)$ to reflect the interaction between neighboring material points:

$$
\tilde{a}(\boldsymbol{X})=\frac{1}{V_{C}} \int_{V_{C}} a(\boldsymbol{y}) \phi(\boldsymbol{y} ; \boldsymbol{X}) \mathrm{d} V,
$$

where $\boldsymbol{y}$ denotes the position in the infinitesimal volume $\mathrm{d} V_{C}$. The weight function $\phi(\boldsymbol{y} ; \boldsymbol{X})$ determines the influence of the local internal variables in this infinitesimal volume on the non-local internal variable at point $\boldsymbol{X}$. For convenience, the weight functions are assumed normalized, such that $\frac{1}{V_{C}} \int_{V_{C}} \phi(\boldsymbol{y} ; \boldsymbol{X}) \mathrm{d} V=1$.

Practically, in order to avoid the direct computation of Eq. (1) in a finite element framework, this expression can be substituted by an explicit gradient enhanced approximated solution as in [59] e.g., or by an implicit form preserving the high order accuracy. In the latter a non-local internal variable is computed through the resolution of a new boundary value problem. Therefore there is no need 
to develop higher-order elements, although the elements have now one additional degree of freedom per node. This approach is fully non-local as it is constructed on the basis of an averaging integral under the form of a new partial differential equation, contrarily to non-local models constructed on the incorporation of higher order terms as for the models of $[60,61]$. This implicit non-local gradient enhanced model has been developed for isotropic materials [29-31]. With a view toward the modeling of the anisotropic behavior of composites, we first extend the gradient-enhanced model to anisotropic materials.

For an anisotropic material, following the same process as for isotropic materials [32], an alternative gradient formulation can be derived in the local coordinates linked to the material system, e.g. linked to the fiber orientation in the unidirectional (UD) continuous fiber-reinforced composite material case. Using the definition of the characteristic length tensor $\boldsymbol{c}_{\mathrm{l}}=\operatorname{diag}\left(c^{i}\right)$, where $c^{i}$ are the squared values of the characteristic length in direction $i$, the Helmholtz-type equation governing the non-local variable is written in the implicit non-local form, see details in Appendix A, as

$$
\tilde{a}-\nabla_{l} \cdot\left(c_{l} \cdot \nabla_{l} \tilde{a}\right)=a,
$$

where " $\nabla_{l}$ " and " $\nabla_{l}$ " represent the gradient operator and the divergence operator expressed in the local coordinates, respectively.

The boundary condition associated with this Helmholtztype equation, which guarantees the equivalence of the volume averages of $\tilde{a}$ and $a$ on the entire problem domain $\Omega$, see for details in Appendix A, reads

$$
\left(\boldsymbol{c}_{1} \cdot \nabla_{l} \tilde{a}\right) \cdot \boldsymbol{n}_{1}=0,
$$

with $\boldsymbol{n}_{1}$ the unit normal to $\Gamma$, the boundary of the entire problem domain $\Omega$, expressed in the local coordinates.

As the material is not always oriented along the global axes, the gradient enhanced formulation should be stated in the inertial frame. A rotation tensor $\boldsymbol{R}$ is defined to represent the change of orthonormal coordinates from the global to the local referential. The anisotropic implicit gradient formulation (2) is thus stated in the global coordinates as

$$
\tilde{a}-\nabla \cdot\left(c_{\mathrm{g}} \cdot \nabla \tilde{a}\right)=a,
$$

where the characteristic length tensor is defined in the global coordinates $\boldsymbol{c}_{\mathrm{g}}=\boldsymbol{R}^{T} \cdot \boldsymbol{c}_{\mathrm{l}} \cdot \boldsymbol{R}$. This equation is completed by the boundary condition (3), which becomes in the global coordinates

$$
\left(\boldsymbol{c}_{\mathrm{g}} \cdot \boldsymbol{\nabla} \tilde{a}\right) \cdot \boldsymbol{n}=0 .
$$

In these last equations " $\nabla$ " and " $\nabla$ " represent the gradient operator and the divergence operator in global coordinates, respectively, and $\boldsymbol{n}$ is the outward unit normal expressed in the global coordinates.

For isotropic materials this tensor reads $c_{\mathrm{g}}=\operatorname{diag}(c)$, with $c=l^{2}$ the square of the characteristic size. For composite materials, the anisotropy can be accounted for in this definition. As an example, for the matrix phase of a unidirectional (UD) continuous fiber-reinforced composite material, $c^{i}=\left(l^{i}\right)^{2}$ is different in the directions parallel and transverse to the fibers: $\boldsymbol{c}_{1}=\operatorname{diag}\left(c^{i}\right)$. The tensor is thus computed from the rotation tensor $\boldsymbol{R}$ describing the fibers orientation.

\subsection{Non-local DG/ECL weak formulation of the laminate problem}

In this section the strong form of the implicit non-local problem within each ply and the strong form governing the delamination of ply interfaces behavior are first recalled. The weak DG/ECL formulation of these strong forms and the resulting finite-element discretization can then be derived.

\subsubsection{Strong formulation of the problem}

Let $\Omega$ be the laminate illustrated in Fig. 1, which is subjected to a force per unit mass $\bar{b}$. Its boundary surface $\Gamma$ includes two parts: the Dirichlet boundary $\Gamma_{D}$ where the displacement is prescribed to $\overline{\overline{\boldsymbol{u}}}$, and the Neumann boundary $\Gamma_{\mathrm{N}}$ where the surface traction is prescribed to $\overline{\overline{\boldsymbol{t}}}$. One has $\Gamma=\Gamma_{D} \cup \Gamma_{T}$ and $\Gamma_{D} \cap \Gamma_{T}=\emptyset$. The body $\Omega$ is divided into several sub-domains of different material behaviors. In this paper the body corresponds to a laminate and the different domains correspond to the different plies with different fiber orientations, Fig. 1, leading to $\Omega=\bigcup_{i} \Omega_{i}$ where $\Omega_{i}$ refers to one material domain with its boundary $\Gamma_{i}$.

The continuum equations thus read

$$
\begin{aligned}
\bar{\rho} \ddot{\overline{\boldsymbol{u}}} & =\boldsymbol{\nabla} \cdot \overline{\boldsymbol{\sigma}}^{T}+\bar{\rho} \overline{\boldsymbol{b}} & & \text { in } \Omega, \\
p & \tilde{p}-\boldsymbol{\nabla} \cdot\left(\boldsymbol{c}_{\mathrm{g}} \cdot \boldsymbol{\nabla} \tilde{p}\right) & & \text { in } \Omega_{i},
\end{aligned}
$$

where $\bar{\rho}$ is the density. The second set of equations results from the implicit non-local Eq. (4) rewritten within each ply $\Omega_{i}$ in terms of the internal variable $p$, which represents the equivalent plastic strain of the composite material matrix, see Section 2.3. As both $p$ and $\tilde{p}$ are related to the matrix phase only, we do not use the over-lined notation. These equations are completed by the following spatial boundary conditions

$$
\begin{aligned}
& \overline{\boldsymbol{u}}=\overline{\overline{\boldsymbol{u}}} \text { on } \Gamma_{\mathrm{D}}, \\
& \overline{\boldsymbol{\sigma}} \cdot \overline{\boldsymbol{n}}=\overline{\overline{\boldsymbol{t}}} \text { on } \Gamma_{\mathrm{N}} \text {, and } \\
& \left(c_{\mathrm{g}} \cdot \boldsymbol{\nabla} \tilde{p}\right) \cdot \overline{\boldsymbol{n}}=0 \quad \text { on } \Gamma .
\end{aligned}
$$

Special attention needs to be paid to the interface $\Gamma_{\mathrm{L}}$ between plies of the laminate. To this end the jump and average operators are defined on an interface lying in the body, which separates the parts arbitrarily denoted $i+1$ and $i$, see Fig. 1, by respectively

$$
\llbracket \bullet \rrbracket=\left[\bullet_{i+1}-\bullet_{i}\right] \text { and }\langle\bullet\rangle=\frac{1}{2}\left[\bullet_{i+1}+\bullet_{i}\right] .
$$

On the un-delaminated part of the interface $\Gamma_{\mathrm{LU}}$, the exact displacement field is continuous with

$$
\llbracket \overline{\boldsymbol{u}}^{\text {exact }} \rrbracket=0 \text { on } \Gamma_{\mathrm{LU}},
$$


where we have considered the jump operator (11). The remaining boundary condition to be defined is related to the non-local field $\tilde{p}$ across these interfaces. A complete discussion on the thermodynamical aspect of this boundary condition can be found in [62], and can be summarized as

- Defining a continuous field $\tilde{p}$ between plies, although not thermodynamically rigorous, results in taking into account the damage process in the neighboring plies of a given ply;

- Using a discontinuous field $\tilde{p}$ between plies corresponds to applying the boundary conditions (10) at the interface between the two materials, restricting direct energy exchanges between plies;

- A boundary condition could be defined to balance the energy exchange across interfaces [62].

As in the problem we are studying, the characteristic dimension is small along the ply thickness, the effect of the boundary conditions between plies is reduced. As tensor $\boldsymbol{c}_{g}$ changes from one ply to another, and as the physical damaging mechanism between plies will be modeled by cohesive zone, for simplicity we choose to apply the boundary conditions at ply interface, i.e. we allow for this field to be discontinuous. One thus has

$$
\left(\boldsymbol{c}_{\mathrm{g}} \cdot \boldsymbol{\nabla} \tilde{p}\right) \cdot \overline{\boldsymbol{n}}=0 \text { on } \Gamma_{\mathrm{LU}_{i+1}} \text { and on } \Gamma_{\mathrm{LU}_{i}} \cdot
$$

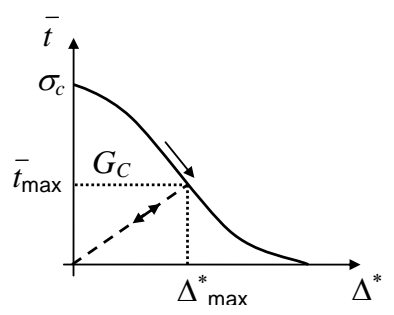

Figure 2: Monotonically decreasing traction separation law (TSL) linking the opening $\boldsymbol{\Delta}^{*}$ to the surface traction $\overline{\boldsymbol{t}}$ and characterized by the strength $\sigma_{c}$, the critical energy release rate $G_{C}$, the maximum opening reached $\boldsymbol{\Delta}_{\max }^{*}$, and the corresponding traction $\overline{\boldsymbol{t}}_{\max }^{*}$. A single arrow refers to the irreversible parts of the TSL and a double arrow refers to the reversible parts.

On the delaminated part $\Gamma_{\mathrm{LC}}$ of the interface $\Gamma_{\mathrm{L}}$ we consider a crack with a traditional traction separation law governing the opening between the crack lips. In terms of the surface traction $\overline{\boldsymbol{t}}=\overline{\boldsymbol{\sigma}} \cdot \overline{\boldsymbol{n}}$ on the crack lips, the governing equations read

$$
\begin{aligned}
& \llbracket \overline{\boldsymbol{t}} \rrbracket=\mathbf{0} \text { on } \Gamma_{\mathrm{LC}}, \\
& \bar{t}=\|\overline{\boldsymbol{t}}\| \leq \bar{t}_{\mathrm{max}} \text { on } \Gamma_{\mathrm{LC}}, \\
& \left(\bar{t}_{\max }-\bar{t}\right) \quad\left\{\begin{array}{cc}
>0 & \text { if } \dot{\Delta}^{*}<0 \text { or } \\
& \Delta^{*}<\Delta_{\text {max }}^{*} ; \\
=0 & \text { if } \dot{\Delta}^{*}>0 \text { and } \\
& \Delta^{*}=\Delta_{\text {max }}^{*}
\end{array}\right. \\
& \left(c_{\mathrm{g}} \cdot \boldsymbol{\nabla} \tilde{p}\right) \cdot \overline{\boldsymbol{n}}=0 \text { on } \Gamma_{\mathrm{LC}_{i+1}} \text { and on } \Gamma_{\mathrm{LC}_{i}} \text {, }
\end{aligned}
$$

where $\bar{t}$ represents the surface traction amplitude between the crack lips, $\Delta^{*}$ is the opening of the crack, and where $\bar{t}_{\max }$ is the surface traction amplitude at the maximum crack opening $\Delta_{\max }^{*}$ reached during the fracture process, see Fig. 2. Equation (17) is similar to Eq. (13) and assumes that once the crack is introduced in the discretization, the crack surfaces act as free boundaries with regards to the non-local implicit equations.

\subsubsection{Weak formulation of the problem}

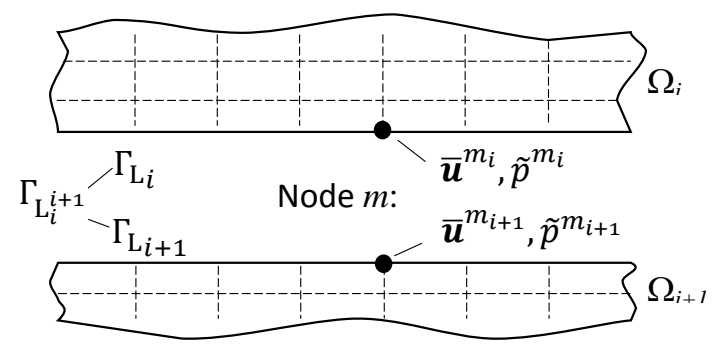

Figure 3: Definition of the degrees of freedom.

The equations governing the strong form are discretized by approximating the unknown fields by continuous approximations in each ply, i.e. $(\overline{\boldsymbol{u}}, \tilde{p}) \in C^{0}\left(\Omega_{i}\right)$, while the fields are discontinuous across the ply interfaces $\Gamma_{\mathrm{L}}$, as illustrated in Fig. 3. Thus the kinematically admissible trial functions $\boldsymbol{w}_{\bar{u}}$ and $w_{\tilde{p}}$, of the displacement and of the non-local fields, respectively, are also discontinuous across the interfaces $\Gamma_{\mathrm{L}}$ which include un-delaminated -or uncracked- surfaces $\Gamma_{\mathrm{LU}}$ on which the strong form (12-13) holds, and delaminated -or cracked surfaces $\Gamma_{\text {LC }}$ on which the strong form (14-17) holds.

The governing equations can be obtained by weakly enforcing the strong form in a weighted-average sense. This is achieved by multiplying the bulk strong form (6-8) by the trial functions $\left(\boldsymbol{w}_{\overline{\boldsymbol{u}}}, w_{\tilde{p}}\right) \in C^{0}\left(\Omega_{i}\right)$, and by integrating by parts on each ply $\Omega_{i}$ instead of performing this integration on the whole domain $\Omega$ as it is usually performed. Using the boundary conditions (8-10, 13 and 17) and definitions (11) of the jump and average operators across the interface $\Gamma_{\mathrm{L}_{i}^{i+1}}$ between the surfaces $\Gamma_{\mathrm{L}_{i}}$ and $\Gamma_{\mathrm{L}_{i+1}}$, see 
Fig. 3, leads to

$$
\begin{aligned}
& \sum_{i} \int_{\Omega_{i}}\left(\bar{\rho} \ddot{\bar{u}} \cdot \boldsymbol{w}_{\bar{u}}+\overline{\boldsymbol{\sigma}}: \nabla \boldsymbol{w}_{\bar{u}}\right) d V+ \\
& \sum_{i} \int_{\Gamma_{\mathrm{LC}_{i}^{i+1}}} \llbracket \boldsymbol{w}_{\bar{u}} \rrbracket \cdot \overline{\boldsymbol{t}}_{i}(\llbracket \overline{\boldsymbol{u}} \rrbracket) d s+ \\
& \sum_{i} \int_{\Gamma_{\mathrm{LU}_{i}^{i+1}}} \llbracket \boldsymbol{w}_{\bar{u}} \rrbracket \cdot\langle\overline{\boldsymbol{\sigma}}\rangle \cdot \overline{\boldsymbol{n}}_{i} d s= \\
& \sum_{i} \int_{\Omega_{i}} \bar{\rho} \overline{\boldsymbol{b}} \cdot \boldsymbol{w}_{\bar{u}} d V+\int_{\Gamma_{\mathrm{N}}} \boldsymbol{w}_{\bar{u}} \cdot \overline{\overline{\boldsymbol{t}}} d s, \\
& \sum_{i} \int_{\Omega_{i}}\left(\tilde{p} w_{\tilde{p}}+\nabla w_{\tilde{p}} \cdot \boldsymbol{c}_{\mathrm{g}} \cdot \boldsymbol{\nabla} \tilde{p}\right) d V= \\
& \sum_{i} \int_{\Omega_{i}} p w_{\tilde{p}} d V
\end{aligned}
$$

where $\overline{\boldsymbol{n}}_{i}$ is the outward unit surface normal of the ply $i$, and where $\overline{\boldsymbol{t}}_{i}$ is the surface traction evaluated at the surface $\Gamma_{\mathrm{L}_{i}}$ of the interface $\Gamma_{\mathrm{L}_{i}^{i+1}}$. Note that as the boundary conditions (10), (13) and (17) have already been used in the derivation of Eq. (19), they do not have to be explicitly applied in the finite element formulation.

The weak formulation (18) does not ensure the continuity of the displacement field at the un-cracked ply interfaces $\Gamma_{\mathrm{LU}}$ and the stability of the method. As usually done with discontinuous Galerkin methods, see [63] e.g. for details, the compatibility equation (12) is enforced in a weak way using the so-called symmetrization terms and sufficiently large quadratic stabilization terms. With the addition of the quadratic terms, the displacement jumps are stabilized in the numerical solution, while the symmetrization terms lead to an optimal convergence rate with respect to the mesh size. The weak formulation (18-19) of the problem is thus finally stated as finding $(\overline{\boldsymbol{u}}, \tilde{p})$ such that

$$
\begin{aligned}
& \sum_{i} \int_{\Omega_{i}}\left(\bar{\rho} \ddot{\overline{\boldsymbol{u}}} \cdot \boldsymbol{w}_{\bar{u}}+\overline{\boldsymbol{\sigma}}: \nabla \boldsymbol{w}_{\bar{u}}\right) d V+ \\
& \sum_{i} \int_{\Gamma_{\mathrm{LC}_{i}^{i+1}}} \llbracket \boldsymbol{w}_{\bar{u} \rrbracket} \rrbracket \cdot \overline{\boldsymbol{t}}_{i}(\llbracket \overline{\boldsymbol{u}} \rrbracket) d s+ \\
& \sum_{i} \int_{\Gamma_{\mathrm{LU}}^{i+1}} \llbracket \boldsymbol{w}_{\bar{u}} \rrbracket \cdot\langle\overline{\boldsymbol{\sigma}}\rangle \cdot \overline{\boldsymbol{n}}_{i} d s+ \\
& \sum_{i} \int_{\Gamma_{\mathrm{LU} i} i+1} \llbracket \overline{\boldsymbol{u}} \rrbracket \cdot\left\langle\overline{\mathbb{C}}_{0}: \nabla \boldsymbol{w}_{\bar{u}}\right\rangle \cdot \overline{\boldsymbol{n}}_{i} d s+ \\
& \sum_{i} \int_{\Gamma_{\mathrm{LU}} i+1} \llbracket \boldsymbol{w}_{\bar{u}} \rrbracket \otimes \overline{\boldsymbol{n}}_{i}:\left\langle\frac{\beta_{s}}{h_{s}} \overline{\mathbb{C}}_{0}\right\rangle: \llbracket \overline{\boldsymbol{u}} \rrbracket \otimes \overline{\boldsymbol{n}}_{i} d s= \\
& \sum_{i} \int_{\Omega_{i}} \bar{\rho} \overline{\boldsymbol{b}} \cdot \boldsymbol{w}_{\bar{u}} d V+\int_{\Gamma_{\mathrm{N}}} \boldsymbol{w}_{\bar{u}} \cdot \overline{\overline{\boldsymbol{t}}} d s, \quad(20) \\
& \int_{\Omega_{i}}\left(\tilde{p} w_{\tilde{p}}+\nabla w_{\tilde{p}} \cdot \boldsymbol{c}_{\mathrm{g}} \cdot \nabla \tilde{p}\right) d V= \\
& \sum_{i} \int_{\Omega_{i}} p w_{\tilde{p}} d V,
\end{aligned}
$$

$\forall\left(\boldsymbol{w}_{\bar{u}}, w_{\tilde{p}}\right)$ kinematically admissible. In this set of equations, $h_{s}$ is the mesh size and $\beta_{s}$ is the penalty parameter for stabilization purpose. In Eq. (20) the initial homogenized elastic operator $\overline{\mathbb{C}}_{0}$ has been used in the symmetrization and stabilization terms instead of the algorithmic operator $\frac{\partial \bar{\sigma}}{\partial \bar{\varepsilon}}$ in order to ensure the stability -which requires non-vanishing quadratic terms- of the method near and beyond the strain softening onset. A complete non-local discontinuous Galerkin formulation accounting for discontinuities within the domain, and not only at the interfaces between the different materials, can be found in [64]. In this formulation the continuity of the non-local field is also weakly ensured across the interfaces.

In the weak form (20-21), the homogenized stress tensor $\overline{\boldsymbol{\sigma}}$ and the homogenized local accumulated plastic strain $p$ are obtained from the damage-enhanced incrementalsecant mean-field homogenization method summarized in Section 2.3. The traction $\overline{\boldsymbol{t}}$ on the delaminated interfaces $\Gamma_{\text {LC }}$ results from the traction separation law developed in Section 2.4. The delamination criterion forcing an initially un-cracked part of $\Gamma_{\mathrm{LU}}$ to become part of the cracked interface $\Gamma_{\mathrm{LC}}$ is also defined in that Section 2.4.

\subsubsection{Finite element implementation}

Each ply $\Omega_{i}$ is discretized into several finite elements $\Omega_{i}^{e}$. The solution of the weak form (20-21) arises by considering polynomial approximations of the displacement mapping $\overline{\boldsymbol{u}}$ and of the non-local accumulated plastic strain $\tilde{p}$, following,

$$
\begin{aligned}
\overline{\boldsymbol{u}}(\boldsymbol{X}) & =\sum_{m} N^{m}(\boldsymbol{X}) \overline{\boldsymbol{u}}^{m}, \\
\boldsymbol{w}_{\bar{u}}(\boldsymbol{X}) & =\sum_{m} N^{m}(\boldsymbol{X}) \delta \overline{\boldsymbol{u}}^{m}, \text { and } \\
\tilde{p}(\boldsymbol{X}) & =\sum_{m} N^{m}(\boldsymbol{X}) \tilde{p}^{m}, \\
\boldsymbol{w}_{\tilde{p}}(\boldsymbol{X}) & =\sum_{m} N^{m}(\boldsymbol{X}) \delta \tilde{p}^{m},
\end{aligned}
$$

where $N^{m}$ is the traditional shape function corresponding to the node $m$. In all generalities, the two interpolations could use different shape functions, but in this paper we consider the same quadratic polynomial approximations for the two fields for simplicity.

As the two fields $\overline{\boldsymbol{u}}$ and $\tilde{p}$ are piece-wise continuous within each ply $\Omega_{i}$ but are discontinuous at the ply interfaces $\Gamma_{\mathrm{L}}$, the shape functions $N$ ought to represent this discontinuity. At each node $m$ of the interface $\Gamma_{\mathrm{L}_{i}^{i+1}}$ the degrees of freedom are duplicated so that the degrees of freedom $\overline{\boldsymbol{u}}^{m_{i}}$ and $\tilde{p}^{m_{i}}$ on ply $i$ coexist with the degrees of freedom $\overline{\boldsymbol{u}}^{m_{i+1}}$ and $\tilde{p}^{m_{i+1}}$ on ply $i+1$, see Fig. 3 .

The finite-element forces can be computed by applying the polynomial approximations (22-23) into the weak form (20-21). Defining $\overline{\mathbf{q}}^{T}=\left[\overline{\mathbf{u}}^{T} \tilde{\mathbf{p}}^{T}\right]$ as the vector regrouping the degrees of freedom of the whole mesh reduces the system into a set of ordinary differential equations, to be 
integrated in the time interval $T$, which are expressed in the matrix form as

$$
\begin{aligned}
\mathbf{M} \ddot{\overline{\mathbf{u}}}+\mathbf{f}_{\overline{\boldsymbol{u}} \text { int }}(\overline{\mathbf{q}})+\mathbf{f}_{\overline{\boldsymbol{u}} \mathrm{L}}(\overline{\mathbf{q}})=\mathbf{f}_{\overline{\boldsymbol{u}} \text { ext }} & \forall t \in T, \\
\mathbf{f}_{\tilde{p} \text { int }}(\overline{\mathbf{q}})-\mathbf{f}_{p \operatorname{int}}(\overline{\mathbf{q}})=0 & \forall t \in T,
\end{aligned}
$$

where $\mathbf{M}$ is the discretized mass matrix, $\mathbf{f}_{\overline{\boldsymbol{u}} \text { int }}$ is the internal forces vector, $\mathbf{f}_{\overline{\boldsymbol{u}} \mathrm{L}}$ is the interface (including the un-delaminated and delaminated parts of $\Gamma_{L}$ ) force vector, $\mathbf{f}_{\overline{\boldsymbol{u}} \text { ext }}$ is the external force vector, $\mathbf{f}_{\tilde{p} \text { int }}$ is the non-local internal force vector related to $\tilde{p}$, and where $\mathbf{f}_{p \text { int }}$ is the non-local force vector related to $p$. These vectors are constructed from the nodal elementary forces reported in Appendix B. The set of equations (24) is completed by the initial conditions $\boldsymbol{u}^{a}(t=0)=0$ and $\dot{\boldsymbol{u}}^{m}(t=0)=\boldsymbol{v}_{0}^{m}$, where $\boldsymbol{v}_{0}^{m}$ are the initial nodal velocities. The time interval of interest $T$ is discretized into time steps and the integration is accomplished through an incremental solution procedure in each time interval $\left[t_{n}, t_{n+1}\right]$.

This DG/ECL finite element formulation

- is consistent, i.e. the exact solution of the problem satisfies equations (20-21);

- is stable if the stability parameter $\beta_{s}$ is larger than a constant that depends on the finite element polynomial order only,

- has the optimal convergence rate with the mesh size $h_{s}$ for linear analyses;

- does not require mesh modification to introduce the crack as a Gauss point of $\Gamma_{\mathrm{LU}}$ simply becomes part of $\Gamma_{\mathrm{LC}}$ when the delamination criterion is met.

The system of equations (24-25) can be solved either in a static way using a Newton-Raphson scheme or in a staggered way in which case equations (24) are solved using an explicit time integration algorithm, while equations (25) are solved using Newton-Raphson iterations. In the latter case, the dynamics Eq. (24) can be integrated using the Hulbert-Chung time integration [65], which exhibits numerical dissipation, and the remaining non-local equations of system (25) are iteratively solved every thousands of explicit steps. Such a staggered technique was previously proposed in [66], and is herein adopted with an implicit resolution of the non-local equations every 5000 explicit steps. The linearized expressions of the non-local equations are reported in Appendix C.

As extensive computations will be required due to the need for fine meshes, the framework is implemented in parallel in Gmsh [67] following the scalable face-based ghost implementation presented in [44, 45] for classical mechanics and adapted in a straightforward way to the non-local implicit scheme.

What remains to be defined are the bulk constitutive material model and the traction-separation law at the cracked interfaces.

\subsection{MFH for elasto-plastic materials with gradient en- hanced damage models}

Instead of using an anisotropic macro-scale material law whose parameters are experimentally identified, we use a mean-field homogenization process, which requires the material parameters of the composite material constituents and the definition of the micro-structures. This method naturally leads to macro-scale anisotropic non-linear behaviors. In our previous work [27, 28], a gradient-enhanced damage multiscale analysis has been developed on the basis of the incremental-secant mean-field homogenization (MFH) [26], and of the gradient-enhanced formulation [32]. In this section after having recalled the non-local damageenhanced J2-elasto-plastic material model of the composite matrix phase, the main ideas of the gradient-enhanced incremental-secant MFH method are given.

\subsubsection{Elasto-plastic materials with non-local enhanced damage models}

The non-local implicit approach recalled in Section 2.1 can be applied to damage models in order to avoid the loss of ellipticity at the macro-scale during the strain-softening.

Damage in the matrix phase is introduced with the usual assumption that the strain tensors in the actual body and in its undamaged representation are equivalent [68, 69]. The usual definition of the effective stress reads

$$
\hat{\boldsymbol{\sigma}}=\frac{\boldsymbol{\sigma}}{(1-D)},
$$

where $\boldsymbol{\sigma}$ is the apparent Cauchy stress and where $0 \leq D<$ 1 is the damage variable.

Assuming an elasto-plastic material for the matrix phase, which obeys J2-elasto-plasticity, the von Mises stress criterion is written in the effective space following

$$
f(\hat{\boldsymbol{\sigma}}, R)=\hat{\sigma}^{\mathrm{eq}}-R(r)-\sigma_{Y} \leq 0
$$

where $f$ is the yield surface, $\hat{\sigma}^{\text {eq }}=\sqrt{\frac{3}{2} \hat{\boldsymbol{\sigma}}: \mathbb{I}^{\mathrm{dev}}: \hat{\boldsymbol{\sigma}}}$ is the equivalent von Mises stress defined using the deviatoric operator $\mathbb{I}^{\mathrm{dev}}, \sigma_{Y}$ is the initial yield stress, and $R(r) \geqslant 0$ is the isotropic hardening stress in terms of $r$, an internal variable related to the accumulated plastic strain $p$ and to the plastic multiplier $\dot{\lambda}$ with $\dot{r}=\dot{\lambda}=(1-D) \dot{p}$, see [70] for details. However in this paper we use the classical approximation that consists in writing the J2-plasticity in the effective stress space: $f(\hat{\boldsymbol{\sigma}}, R(p)) \leqslant 0$. During the plastic flow, i.e. $f=0, \Delta p>0$, the plastic strain tensor increment follows the plastic flow direction

$$
\Delta \varepsilon^{\mathrm{p}}=\Delta p \frac{\partial f}{\partial \hat{\boldsymbol{\sigma}}}=\frac{3 \Delta p}{2} \frac{\mathbb{I}^{\mathrm{dev}}: \hat{\boldsymbol{\sigma}}}{\hat{\boldsymbol{\sigma}}^{\mathrm{eq}}}=\Delta p \boldsymbol{N}
$$

where $\boldsymbol{N}$ is the normal to the yield surface in the effective stress space. The apparent stress follows from the coupled damage concept and reads

$$
\boldsymbol{\sigma}=(1-D) \mathbb{C}^{\mathrm{el}}:\left(\varepsilon-\varepsilon^{\mathrm{p}}\right)=(1-D) \mathbb{C}^{\mathrm{el}}: \boldsymbol{\varepsilon}^{\mathrm{el}} .
$$


This formulation is completed by the damage evolution description. In this work the non-local accumulated plastic strain $\tilde{p}$ is applied to calculate the damage evolution in the incremental damage model:

$$
\Delta D= \begin{cases}0, & \text { if } \tilde{p} \leqslant p_{C} \\ \left(\frac{Y_{n+\alpha}}{S_{0}}\right)^{s} \Delta \tilde{p}, & \text { if } \tilde{p}>p_{C} .\end{cases}
$$

In this expression, $p_{C}$ is a plastic threshold for the damage evolution, $S_{0}$ and $s$ are the material parameters, $Y$ is the strain energy release rate computed as

$$
Y=\frac{1}{2} \varepsilon^{\mathrm{el}}: \mathbb{C}^{\mathrm{el}}: \varepsilon^{\mathrm{el}},
$$

and $\alpha$ is an interpolation parameter ranging from 0 to 1 .

In the non-local implicit approach the non-local accumulated plastic strain $\tilde{p}$ is computed from the implicit formulation (4), which is rewritten

$$
\tilde{p}-\nabla \cdot\left(\boldsymbol{c}_{\mathrm{g}} \cdot \nabla \tilde{p}\right)=p .
$$

where $\boldsymbol{c}_{\mathrm{g}}$ is the characteristic squared lengths tensor as defined in Section 2.1.

\subsubsection{Incremental-secant mean-field homogenization for two-phase composites}

In the multiscale approach depicted in Fig. 1, at each macro-point $\boldsymbol{X}$ of a ply, the macro-strain tensor $\bar{\varepsilon}$ is known, and the macro-stress tensor $\overline{\boldsymbol{\sigma}}$ is sought from the resolution of a micro-scale boundary value problem (BVP). The Hill-Mandell condition, expressing the equality between energies at both scales, transforms the relation between macro-strains $\bar{\varepsilon}$ and stresses $\overline{\boldsymbol{\sigma}}$ into the relation between the volume average strains $\langle\varepsilon\rangle_{\omega}$ and stresses $\langle\boldsymbol{\sigma}\rangle_{\omega}$ over the RVE. For a two-phase isothermal composite with the respective volume fractions $v_{0}+v_{\mathrm{I}}=1$ (subscript 0 refers to the matrix and I to the inclusions), the average quantities are expressed in terms of the phase averages as

$$
\overline{\boldsymbol{\varepsilon}}=v_{0}\langle\boldsymbol{\varepsilon}\rangle_{\omega_{0}}+v_{\mathrm{I}}\langle\varepsilon\rangle_{\omega_{\mathrm{I}}} \quad \text { and } \quad \overline{\boldsymbol{\sigma}}=v_{0}\langle\boldsymbol{\sigma}\rangle_{\omega_{0}}+v_{\mathrm{I}}\langle\boldsymbol{\sigma}\rangle_{\omega_{\mathrm{I}}}
$$

For simplicity, in the following developments, the notations $\langle\bullet\rangle_{\omega_{i}}$ will be replaced by $\bullet_{i}$. Considering the socalled linear comparison composite (LCC), the relation between the average incremental strains in the two phases depends on the chosen expressions of the virtual elastic operators $\mathbb{C}_{0}^{\mathrm{LCC}}$ of the matrix phase and $\mathbb{C}_{\mathrm{I}}^{\mathrm{LCC}}$ of the inclusions phase, leading to

$$
\Delta \varepsilon_{\mathrm{I}}=\mathbb{B}^{\epsilon}\left(\mathrm{I}, \mathbb{C}_{0}^{\mathrm{LCC}}, \mathbb{C}_{\mathrm{I}}^{\mathrm{LCC}}\right): \Delta \varepsilon_{0} .
$$

On the one hand, for a single ellipsoidal inclusion $\mathbb{B}^{\epsilon}$ in a infinite matrix can be obtained from the Eshelby solution [13]. On the other hand, in the case of multiple inclusions $\mathbb{B}^{\epsilon}$ can be obtained analytically under some assumptions on the micro-mechanics $[14,17]$. In this paper, the Mori-Tanaka (M-T) expression of $\mathbb{B}^{\epsilon}$, which assumes that the strain at infinity in the single inclusion problem corresponds to the average strain in the matrix phase, is used and the strain concentration tensor reads

$$
\mathbb{B}^{\epsilon}=\left\{\mathbb{I}+\mathbb{S}:\left[\left(\mathbb{C}_{0}^{\mathrm{LCC}}\right)^{-1}: \mathbb{C}_{\mathrm{I}}^{\mathrm{LCC}}-\mathbb{I}\right]\right\}^{-1},
$$

where the Eshelby tensor $\mathbb{S}\left(\mathrm{I}, \mathbb{C}_{0}^{\mathrm{LCC}}\right)$ depends on the geometry of the inclusion (I) and on the virtual elastic operator $\mathbb{C}_{0}^{\mathrm{LCC}}$. The expressions of the tensors $\mathbb{C}_{0}^{\mathrm{LCC}}$ and $\mathbb{C}_{\mathrm{I}}^{\mathrm{LCC}}$ depend on the chosen $\mathrm{MFH}$ process. In linear elasticity, these operators correspond to the material moduli $\mathbb{C}_{0}^{\mathrm{el}}$ and $\mathbb{C}_{\mathrm{I}}^{\mathrm{el}}$. In the incremental-tangent $\mathrm{MFH}$ method for non-linear materials, they correspond to the "consistent" average tangential operators $\mathbb{C}_{0}^{\text {alg }}$ and $\mathbb{C}_{\mathrm{I}}^{\text {alg }}$, and are uniform by construction. Although this incremental-tangent method can be used for elasto-plastic constitutive materials exhibiting damage [27], such an approach implicitly implies -through the linearization of (34) - that the strain rates in both phases have the same sign. However for composite materials, during the strain softening of the matrix (positive strain rate) the fibers can see an elastic unloading (negative strain rate). This has motivated the development of the incremental-secant method $[26,28]$ to be able to capture this behavior. Moreover within the incremental-secant formalism, the new secant operator of the matrix phase, $\mathbb{C}_{0}^{\mathrm{S}}$ is naturally isotropic, which prevents the isotropisation step required with the incremental-tangent method [22].

Considering a time interval $\left[t_{n}, t_{n+1}\right]$, with the total strain tensor $\bar{\varepsilon}_{n}$ at time $t_{n}$ and the strain increment $\Delta \bar{\varepsilon}_{n+1}$ resulting from the $\mathrm{FE}$ resolution, the strain tensor $\bar{\varepsilon}_{n+1}$ at time $t_{n+1}$ follows from

$$
\bar{\varepsilon}_{n+1}=\bar{\varepsilon}_{n}+\Delta \bar{\varepsilon}_{n+1} .
$$

At time $t_{n}$, a virtual elastic unloading from the stress state $\overline{\boldsymbol{\sigma}}_{n}$ is applied, which corresponds to a residual strain tensor $\bar{\varepsilon}_{n}^{\text {res }}$, see Fig. 4(a). The unloading $\Delta \bar{\varepsilon}_{n}^{\text {unload }}$ is chosen so that the homogenized residual stress of the composite material vanishes

$$
\overline{\boldsymbol{\sigma}}_{n}^{\text {res }}=v_{0} \boldsymbol{\sigma}_{0}^{\text {res }}+v_{\mathrm{I}} \boldsymbol{\sigma}_{\mathrm{I}}^{\text {res }}=0 .
$$

The main idea of the incremental-secant method, is to define a LCC subjected to a strain increment $\Delta \bar{\varepsilon}_{n+1}^{\mathrm{r}}$ from the virtually unloaded state, satisfying

$$
\bar{\varepsilon}_{n+1}=\bar{\varepsilon}_{n}^{\mathrm{res}}+\Delta \bar{\varepsilon}_{n+1}^{\mathrm{r}},
$$

and from which the stress tensor at time $t_{n+1}$ can be computed. The resulting stress and strain states in the composite material are illustrated in Fig. 4(a).

To complete the incremental-secant MFH process, the residual stress and strain state in the phases of the composite material illustrated in Fig. 4(b) have to be defined. At time $t_{n+1}$ the stress and damage values reach respectively $\boldsymbol{\sigma}_{n+1}$ and $D_{n+1}$. Following the method pictured on Fig. 4(b), the effective stress tensor at time $t_{n+1}$ can also be expressed as

$$
\hat{\boldsymbol{\sigma}}_{n+1}=\hat{\boldsymbol{\sigma}}_{n}^{\mathrm{res}}+\Delta \hat{\boldsymbol{\sigma}}_{n+1}^{\mathrm{r}}, \text { with } \Delta \hat{\boldsymbol{\sigma}}_{n+1}^{\mathrm{r}}=\mathbb{C}^{\mathrm{Sr}}: \Delta \varepsilon_{n+1}^{\mathrm{r}} .
$$




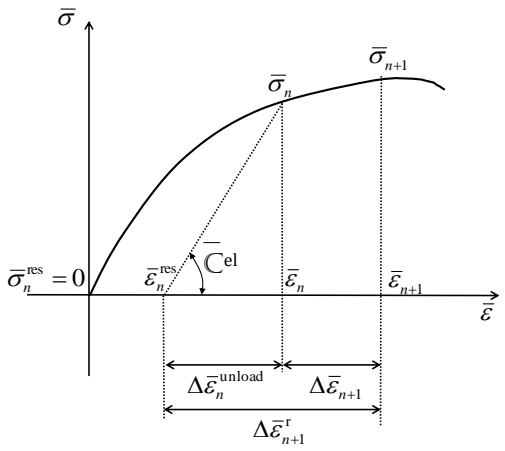

(a) Composite

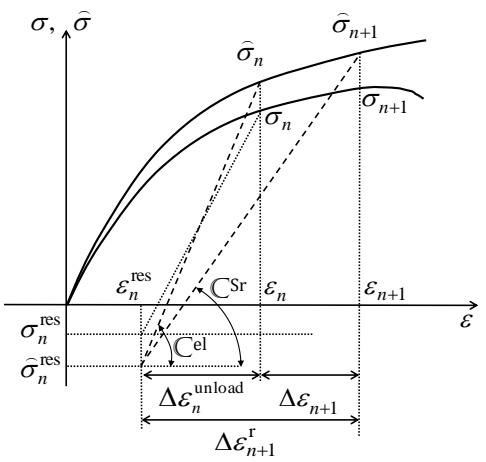

(b) Residual-secant

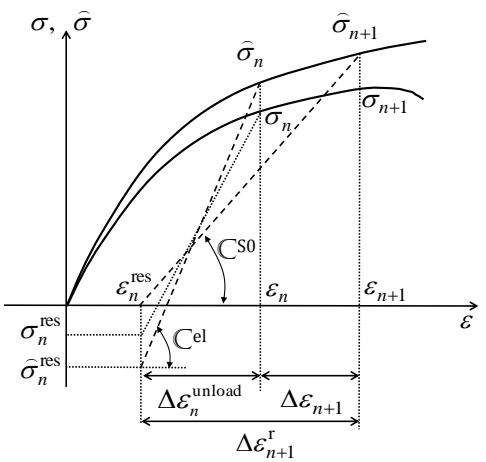

(c) Zero-secant

Figure 4: Definition of the incremental-secant formulation [28]. (a) Virtual unloading and incremental-secant formulation on the composite material. (b) Definition of the residual strain and stress and of the residual-secant operator in a phase. (c) Definition of the zerosecant operator in a phase.

In this last equation $\mathbb{C}^{\mathrm{Sr}}$ is the residual-incremental-secant operator of the undamaged linear comparison material. In particular for J2-elasto-plastic materials this operator is isotropic and can be expressed as

$$
\mathbb{C}^{\mathrm{Sr}}=3 \kappa^{\mathrm{r}} \mathbb{I}^{\mathrm{vol}}+2 \mu_{s}^{\mathrm{r}} \mathbb{I}^{\mathrm{dev}}
$$

with the equivalent undamaged bulk and shear moduli $\kappa^{\mathrm{r}}$ and $\mu_{s}^{\mathrm{r}}$ that can be computed following [28].

Finally the apparent stress tensor is readily obtained from

$$
\boldsymbol{\sigma}_{n+1}=\left(1-D_{n+1}\right) \hat{\boldsymbol{\sigma}}_{n+1}
$$

and the residual-incremental-secant operator of the damaged isotropic-linear comparison material can directly be evaluated from

$$
\mathbb{C}^{\mathrm{SDr}}=\left(1-D_{n+1}\right) \mathbb{C}^{\mathrm{Sr}}=3 \kappa^{\mathrm{Dr}} \mathbb{I}^{\mathrm{vol}}+2 \mu_{s}^{\mathrm{Dr}} \mathbb{I}^{\mathrm{dev}},
$$

with the equivalent damaged bulk and shear elastic moduli $\kappa^{\mathrm{Dr}}$ and $\mu_{s}^{\mathrm{Dr}}$ that can be computed following [28]

As lengthy discussed in [26], when defining the LCC, it can be advantageous to modify the residual-incrementalsecant approach by neglecting the residual stress -but not the residual strain- in the matrix phase for composites whose inclusions remain elastic, or exhibit an elasto-plastic behavior with a hardening coefficient higher than the one of the elasto-plastic matrix material.

The modification follows the suggestion illustrated in Fig. 4(c) and consists in neglecting $\hat{\boldsymbol{\sigma}}_{n}^{\text {res }}$ in the formalism described here above, which leads to defining the two operators

$$
\begin{aligned}
\mathbb{C}^{\mathrm{S} 0} & =3 \kappa^{0} \mathbb{I}^{\mathrm{vol}}+2 \mu_{s}^{0} \mathbb{I}^{\mathrm{dev}}, \text { and } \\
\mathbb{C}^{\mathrm{SD} 0} & =\left(1-D_{n+1}\right) \mathbb{C}^{\mathrm{S} 0}=3 \kappa^{\mathrm{D} 0} \mathbb{I}^{\mathrm{vol}}+2 \mu_{s}^{\mathrm{D} 0} \mathbb{I}^{\mathrm{dev}}
\end{aligned}
$$

respectively the zero-incremental-secant operator of the un-damaged isotropic-linear comparison material and the zero-incremental-secant operator of the damaged isotropiclinear comparison material.

The MFH scheme can now be applied from the unloaded state. The MFH scheme stated by Eqs. (33-35) is finally rewritten as

$$
\begin{aligned}
\Delta \bar{\varepsilon}_{n+1}^{\mathrm{r}} & =v_{0} \Delta \varepsilon_{0 n+1}^{\mathrm{r}}+v_{\mathrm{I}} \Delta \varepsilon_{\mathrm{I} n+1}^{\mathrm{r}}, \\
\overline{\boldsymbol{\sigma}}_{n+1} & =v_{0} \boldsymbol{\sigma}_{0 n+1}+v_{\mathrm{I}} \boldsymbol{\sigma}_{\mathrm{I} n+1}, \\
\Delta \varepsilon_{\mathrm{I} n+1}^{\mathrm{r}} & =\mathbb{B}^{\epsilon}\left(\mathrm{I}, \mathbb{C}_{0}^{\mathrm{SD} 0}, \mathbb{C}_{\mathrm{I}}^{\mathrm{Sr}}\right): \Delta \varepsilon_{0 n+1}^{\mathrm{r}},
\end{aligned}
$$

where in this last equation we have considered a matrix phase experiencing damage, hence the use of the zeroincremental-secant operator $\mathbb{C}_{0}^{\mathrm{SD} 0}$ of the equivalent damage material, and an inclusion phase experiencing no damage, hence the use of the residual-incremental-secant operator $\mathbb{C}_{\mathrm{I}}^{\mathrm{Sr}}$ of the equivalent material The resolution of the set of Eqs. (44-46) follows the iterative process described in [28].

Note: the local variable $p$ and the non-local variable $\tilde{p}$ are uniform representation of the local matrix accumulated plastic strain $p_{0}$ and of its non-local value $\tilde{p}_{0}$, but they do not correspond to their mean values in the matrix.

\subsection{Traction separation law (TSL)}

The delamination process is usually modeled by recourse to cohesive elements which integrate an intrinsic cohesive law, see [33-38] among many others. In that case, the TSL of the cohesive element also models the elastic response prior to the delamination process, yielding mesh-size effect $[37,38]$.

In the presented DG/ECL framework, the response prior to delamination is accounted for by the DG formalism, avoiding this mesh-size effect. Moreover this formalism 


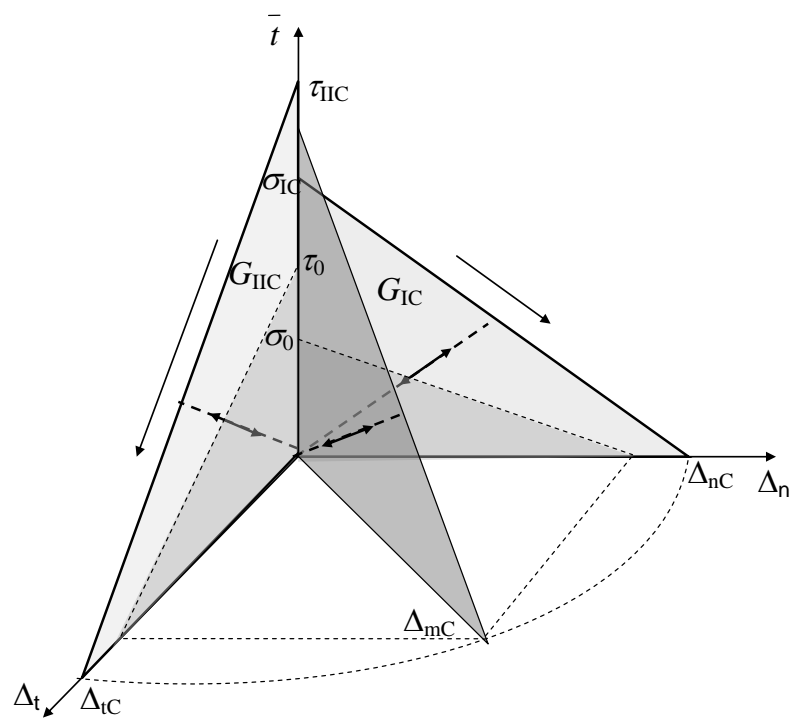

Figure 5: Mixed mode traction separation law.

virtually allows for any constitutive material behavior to be considered within the ply while the stresses at ply interfaces remain accessible in order to evaluate a delamination criterion such as the one proposed in [36]. However, because of the existence of the damaging process taking place in the matrix phase of the ply, the stresses are reduced in the presence of damage $D$, which could prevent the delamination initiation. In this paper we propose to use the following criterion

$$
\frac{\ll \sigma \gg^{2}}{\sigma_{\text {IC }}^{2}}+\frac{\tau^{2}}{\tau_{\text {II C }}^{2}} \leq(1-D)^{2}
$$

where $\sigma=\boldsymbol{n} \cdot \boldsymbol{\sigma} \cdot \boldsymbol{n}$ and $\tau=\sqrt{(\boldsymbol{n} \cdot \boldsymbol{\sigma}) \cdot(\boldsymbol{n} \cdot \boldsymbol{\sigma})-\sigma^{2}}$ are respectively the normal and tangent components of the apparent surface traction $\overline{\boldsymbol{t}}$ at the interface, $\sigma_{\mathrm{IC}}$ and $\tau_{\mathrm{IIC}}$ are the maximum tension and shearing of the cohesive model, and where the operator $\ll \bullet \gg$ refers to the positive value, i.e. equals to zero in case of negative argument. At delamination initiation, the respective (apparent) stress components are referred to as $\sigma_{0}$ and $\tau_{0}$, see Fig. 5 .

During the delamination process, the opening and shearing between the two plies respectively result into the energy release rates $G_{\mathrm{I}}$ and $G_{\mathrm{II}}$, with a complete fracture obtained for

$$
\left(\frac{G_{\mathrm{I}}}{G_{\mathrm{IC}}}\right)^{\alpha}+\left(\frac{G_{\mathrm{II}}}{G_{\mathrm{IIC}}}\right)^{\alpha}=1,
$$

where $G_{\text {IC }}$ and $G_{\text {II C }}$ are the mode I and mode II critical energy release rates respectively, and where $\alpha$ is a mixed mode parameter. The critical openings for pure mode I and mode II fracture are respectively obtained from $\Delta_{\mathrm{IC}}=$ $\frac{2 G_{\mathrm{I}}}{\sigma_{\mathrm{IC}}}$ and from $\Delta_{\mathrm{IIC}}=\frac{2 G_{\mathrm{IIC}}}{\tau_{\mathrm{IIC}}}$.

To model a mixed-mode opening, see Fig. 5, an effective surface separation $\Delta^{*}$ is evaluated from the surface opening vector $\llbracket \overline{\boldsymbol{u}} \rrbracket$ by

$$
\Delta^{*}=\sqrt{\ll \Delta_{\mathrm{n}} \gg^{2}+\Delta_{\mathrm{t}}^{2}},
$$

where $\Delta_{\mathrm{n}}=\llbracket \overline{\boldsymbol{u}} \rrbracket \cdot \boldsymbol{n}$ is the separation along the interface element normal $\boldsymbol{n}$, and where $\Delta_{\mathrm{t}}=\llbracket \overline{\boldsymbol{u}} \rrbracket-\Delta_{\mathrm{n}} \boldsymbol{n}$ is the separation along the interface element tangent $\overline{\boldsymbol{t}}=\frac{\llbracket \overline{\boldsymbol{u}} \rrbracket-\Delta_{\mathrm{n}} \boldsymbol{n}}{\left\|\llbracket \overline{\boldsymbol{u}} \rrbracket-\Delta_{\mathrm{n}} \boldsymbol{n}\right\|}$. This definition of the effective opening is completed by

$$
\beta= \begin{cases}\frac{\tau}{\sigma} & \text { if } \Delta_{\mathrm{n}}>0 \\ 0 & \text { if } \Delta_{\mathrm{n}} \leq 0\end{cases}
$$

which characterizes the failure mode, and by

$$
\Delta_{\max }=\max _{t^{\prime} \leq t}\left(\Delta^{*}\left(t^{\prime}\right)\right)
$$

the maximum opening reached up to the current time $t$.

These definitions allow defining the critical mixed mode opening $\Delta_{\mathrm{mC}}$ that $\Delta^{*}$ can reach to release the correct amount of energy for a constant $\beta^{1}[36]$

$$
\Delta_{\mathrm{mC}}= \begin{cases}\frac{2\left(1+\beta^{2}\right)}{\sqrt{\ll \sigma_{0} \gg^{2}+\tau_{0}^{2}}}\left[\frac{1}{G_{\mathrm{IC}}^{\alpha}}+\frac{\beta^{2 \alpha}}{G_{\mathrm{IIC}}^{\alpha}}\right]^{-\frac{1}{\alpha}} & \text { if } \Delta_{\mathrm{n}}>0, \\ \Delta_{\mathrm{IIC}} & \text { if } \Delta_{\mathrm{n}} \leq 0 .\end{cases}
$$

The traction separation law directly follows from

$$
\begin{gathered}
\bar{\sigma}=\left\{\begin{array}{lr}
\ll \sigma_{0} \gg\left(1-\frac{\Delta^{*}}{\Delta_{\mathrm{mC}}}\right) & \text { if } \dot{\Delta}^{*} \geqslant 0 \text { and } \\
\bar{\sigma}_{\max } \frac{\Delta^{*}}{\Delta_{\max }} & \text { if } \dot{\Delta}^{*}<0,
\end{array}\right. \\
\bar{\tau}=\left\{\begin{array}{lr}
\tau_{0}\left(1-\frac{\Delta^{*}}{\Delta_{\mathrm{mC}}}\right) & \text { if } \dot{\Delta}^{*} \geqslant 0 \text { and } \\
\Delta^{*}=\Delta_{\max }, \\
\bar{\tau}_{\max \frac{\Delta^{*}}{\Delta_{\max }}} & \text { if } \dot{\Delta}^{*}<0,
\end{array}\right.
\end{gathered}
$$

where $\bar{\sigma}$ and $\bar{\tau}$ are the components of the tractionseparation law $\overline{\boldsymbol{t}}$

$$
\overline{\boldsymbol{t}}=\bar{\sigma} \boldsymbol{n}+\bar{\tau} \boldsymbol{t},
$$

and where $\bar{\sigma}_{\max }$ and $\bar{\tau}_{\max }$ correspond to the normal and tangential components, respectively, reached at maximum opening $\Delta_{\max }$. Indeed the linear cohesive law, as shown in Fig. 5, includes an irreversible softening part during the crack opening and a reversible part if a crack unloading occurs.

\section{Numerical simulations and validations}

First the required material parameters of carbon fibers and of the epoxy matrix of prepreg Hexply M10.1/38\% / UD300 / HS (R) are identified from the manufacturer

\footnotetext{
${ }^{1}$ During the delamination process, the relative contributions of modes I and II could vary, as illustrated in e.g. [9], in which case a damage-based thermodynamically consistent cohesive model, such as the one proposed in [37], should be used.
} 
data. The cohesive energy required for the delamination model is extracted from a Double Cantilever Beam (DCB) experiment. Using these material parameters, the model behavior is first studied by comparing the results obtained from the anisotropic gradient-enhanced MFH model to the results obtained with the isotropic gradient-enhanced MFH model. Afterward, the model is validated by comparing numerical predictions to experimental tensile tests on open-hole laminates with different stacking sequences.

\subsection{Materials identifications}

Table 1: Material properties of the carbon fibers.

\begin{tabular}{ll}
\hline Property & Value \\
\hline Long. Young's modulus $E_{\mathrm{L}}[\mathrm{GPa}]$ & 230 \\
Trans. Young's modulus $E_{\mathrm{T}}[\mathrm{GPa}]$ & 40 \\
Trans. Poisson ratio $\nu_{\mathrm{TT}}[-]$ & 0.20 \\
Long.-Trans. Poisson ratio $\nu_{\mathrm{LT}}[-]$ & 0.256 \\
Trans. shear modulus $G_{\mathrm{TT}}[\mathrm{GPa}]$ & 16.7 \\
Long.-Trans. shear modulus $G_{\mathrm{LT}}[\mathrm{GPa}]$ & 24 \\
\hline
\end{tabular}

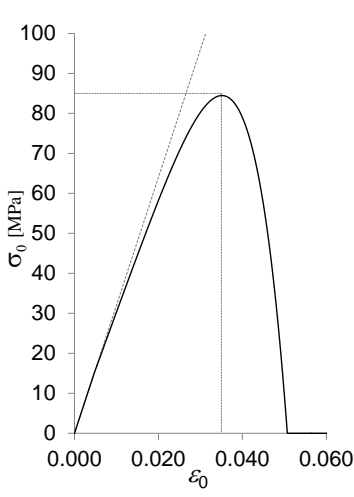

(a) Stress

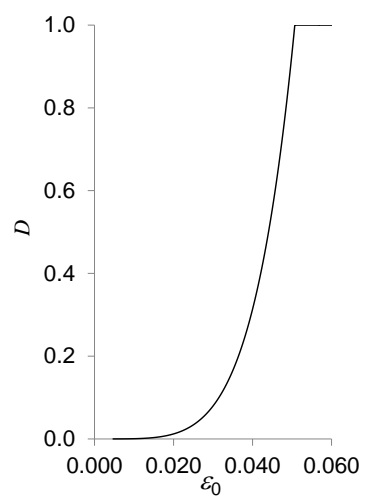

(b) Damage
Figure 6: Idealized material law of the epoxy matrix in tension.

Table 2: Material properties of the epoxy.

\begin{tabular}{ll}
\hline Property & Value \\
\hline Young's modulus $E_{0}[\mathrm{GPa}]$ & 3.2 \\
Poisson ratio $\nu_{0}[-]$ & 0.3 \\
Initial yield stress $\sigma_{Y_{0}}[\mathrm{MPa}]$ & 15 \\
Hardening modulus $h_{0}[\mathrm{MPa}]$ & 300 \\
Hardening exponent $m_{0}[-]$ & 100 \\
Damage critical energy release $S_{0}[\mathrm{Mpa}]$ & 0.1 \\
Damage exponent $s_{0}[-]$ & 1.73 \\
Damage critical plastic strain $p_{C}[-]$ & 0.0 \\
\hline
\end{tabular}

The material studied in this section is a continuous carbon fibers reinforced epoxy composite. The material is made of prepreg Hexply M10.1/38\% / UD300 / HS (R), which results in a fiber volume fraction of $60 \%$ after curing $^{2}$. When manufacturing the samples, the prepreg was cured at $120{ }^{\circ} \mathrm{C}$ during $60 \mathrm{~min}$ under an applied pressure of $0.4 \mathrm{MPa}$.

The carbon fibers are assumed to be linear elastic and transversely isotropic. Typical material constants, see e.g [71], for carbon fibers are considered and are reported in Table 1.

The cured epoxy matrix properties reported by the manufacturer are a tensile modulus of $3.2 \mathrm{GPa}$, and a tensile strength of $85 \mathrm{MPa}$ at 0.035 strain. By lack of elastoplastic data, an exponential hardening law

$$
R_{0}(p)=h_{0}\left[1-e^{-m_{0} p}\right]
$$

and a damage law obeying to Eq. (30) are defined, with the material properties reported in Table 2. The corresponding stress-strain curve of the epoxy matrix is illustrated on Fig. 6(a) and the manufacturer data are also reported. The parameters of the matrix law were defined so that the maximum experimental strength and strain are captured by the material model. From the damage evolution on Fig. $6(\mathrm{~b})$, it can be seen that softening onset is reached for a strain around 0.032 . The real behavior of an epoxy resin is more complex as it experiences different responses in compression and tension. However, as the simulations will be carried out under tension, we only consider the tensile strength of the material in this idealized curve.

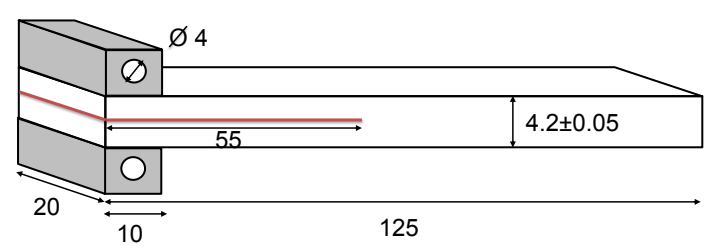

Figure 7: DCB setup, units in mm.

In order to characterize the traction separation law of the cohesive model, a delamination test on a Double Cantilever Beam (DCB), is performed following the ISO15024-norm. The specimens were cut from an autoclave consolidated unidirectional laminate panel at $0^{\circ}$, with an initially 55-mm-long delaminated zone obtained using adhesive. Two loading blocks were glued on the sample delaminated extremity, see Fig. 7.

The sample is first loaded up to crack initiation before being unloaded. When applying this loading $P$ on the

\footnotetext{
${ }^{2}$ The percentage is obtained from a microscopic imaging process of the cured laminates
} 


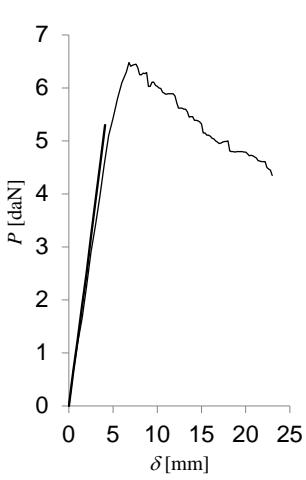

(a) Loading

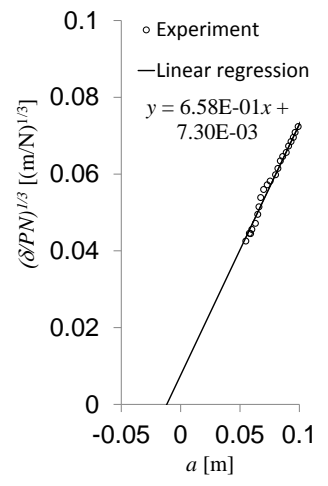

(b) Compliance

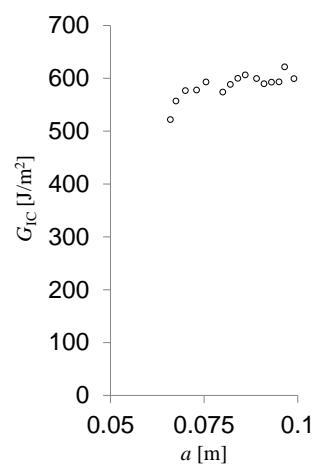

(c) Energy release rate

Figure 8: Experimental DCB test.

Table 3: Material properties of the delamination model.

\begin{tabular}{ll}
\hline Property & Value \\
\hline Mode I critical energy release rate $G_{\mathrm{IC}}\left[\mathrm{J} / \mathrm{m}^{2}\right]$ & 600 \\
Mode II critical energy release rate $G_{\mathrm{IIC}}\left[\mathrm{J} / \mathrm{m}^{2}\right]$ & 1200 \\
Mode I critical stress $\sigma_{\mathrm{IC}}[\mathrm{MPa}]$ & 20 \\
Mode II critical stress $\tau_{\mathrm{II}}[\mathrm{MPa}]$ & 20 \\
Mixed mode parameter $\alpha[-]$ & 1 \\
\hline
\end{tabular}

blocks, the evolution of the opening $\delta$ at loading point and the evolution of the delamination length $a$ are recorded. The evolution of the opening in terms of the loading is reported in Fig. 8(a). The regression analysis of the modified compliance $\delta /(P N)$, where $N$ is a correction factor defined by the norm to account for the block loading, in terms of the crack propagation $a$ is reported in Fig. 8(b). This regression analysis leads to the definition of $\Delta=-0.01162$ $\mathrm{m}$, the extrapolated negative crack size corresponding to a zero compliance. This value is required by the norm to evaluate the critical energy release rate. The norm can then be used to define the corresponding critical energy release rate following $G_{\mathrm{IC}}=\frac{3 P \delta}{2 b(a+|\Delta|)} \times \frac{F}{N}$, where $b$ is the sample width and where $F / N \simeq 1$ is a correcting factor given by the norm. The results obtained for a compliance at least $5 \%$ higher than the original one (the $5 \%$ value of the norm) are reported in Fig. 8(c). It can be seen that during the crack propagation a value of $G_{\mathrm{IC}} \simeq 600 \mathrm{~J} / \mathrm{m}^{2}$ can be considered. For mode II, the critical energy for carbon-fiber reinforced composite material is usually several times higher than for mode I and a value twice higher is assumed herein by lack of mode II delamination tests. The critical stresses $\sigma_{\mathrm{IC}}$ and $\tau_{\mathrm{IIC}}$ are taken equal to 20 $\mathrm{MPa}$ so that the critical openings $\Delta_{\mathrm{IC}}=\frac{2 G_{\mathrm{IC}}}{\sigma_{\mathrm{IC}}}=0.06$ $\mathrm{mm}$ and $\Delta_{\mathrm{IIC}}=\frac{2 G_{\mathrm{IIC}}}{\tau_{\text {II }}}=0.12 \mathrm{~mm}$ are of comparable size with the distance between Gauss points at the interface elements. As pointed out in $[38,54]$, in order to initiate the delamination, the element size should be small enough to resolve the cohesive model. In order to keep reasonable element sizes while ensuring the correct propagation of the delaminated zone, the critical stress can be lowered as long as the proper energy is dissipated, which is the case by considering the correct values of $G_{\mathrm{IC}}$ and $G_{\mathrm{IIC}}$. The complete parameters list of the cohesive law is reported in Table 3.

Before validating the model with experiments, the effect of the characteristic length is first described.

\subsection{Effect of the anisotropic gradient model}

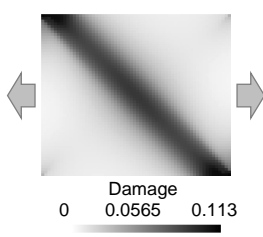

(a) $c^{2}=2.0 \mathrm{~mm}^{2}$

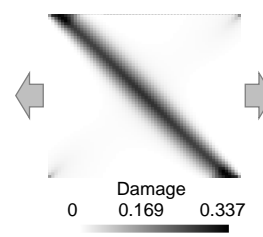

(b) $c^{2}=8.45 \times 10^{-5}$ $\mathrm{mm}^{2}$

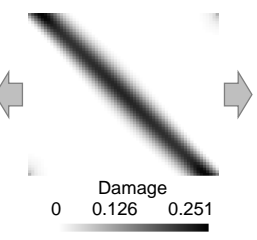

(c) $c^{1}=c^{2}=8.45 \times$ $10^{-5} \mathrm{~mm}^{2}$ and $c^{3}=$ $2.0 \mathrm{~mm}^{2}$
Figure 9: Damage distribution in a $45^{\circ}$ unidirectional ply under tensile loading. Comparison of results for (a) The isotropic gradientenhanced model with $c^{2}=2.0 \mathrm{~mm}^{2}$, (b) The isotropic gradientenhanced model with $c^{2}=8.45 \times 10^{-5} \mathrm{~mm}^{2}$, and (c) The anisotropic gradient-enhanced model with $c^{1}=c^{2}=8.45 \times 10^{-5} \mathrm{~mm}^{2}$ and $c^{3}=2.0 \mathrm{~mm}^{2}$. The arrows represent the loading direction.

The model is a rectangular composite plate under tensile loading. The sizes of the plates are $10 \mathrm{~mm}$ (along the loading direction) $\times 8.5 \mathrm{~mm}$ (perpendicular to the loading direction). The mesh size is about $0.2 \mathrm{~mm}$. The test is $3 \mathrm{D}$ with plane stress state along the thickness. In this composite plate, all the fibers are set to be along the $45^{\circ}$ direction. Due to the geometry and the boundary conditions of the problem, the damage will initiate at the boundary of the plate.

The numerical simulations are carried out successively with the isotropic gradient-enhanced MFH model and with the improved anisotropic gradient-enhanced MFH model developed herein. For the isotropic gradient model, two different characteristic length parameters $c^{2}$, see Section 2.1, are considered: the first one is $c^{2}=2.0 \mathrm{~mm}^{2}$ according to the reference [72], and the second one is $c^{2}=8.45 \times 10^{-5}$ $\mathrm{mm}^{2}$, which is chosen according to the distance between 
the fibers. For the anisotropic gradient model, $c^{1}=c^{2}=$ $8.45 \times 10^{-5} \mathrm{~mm}^{2}$ are used for the transverse directions to the fibers, and $c^{3}=2.0 \mathrm{~mm}^{2}$ for the longitudinal direction of the fibers. Indeed, for a real material, on the one hand, fibers have the effect of preventing the interactions among the matrix material points in the transverse direction, and on the other hand, they dominate the long range effects in the longitudinal direction [49].

The damage distributions obtained by the different models are presented in Fig. 9 for a tensile displacement equal to $0.3 \mathrm{~mm}$. From the damage distribution, we can see that for the isotropic gradient enhanced MFH model, when the large characteristic length is used, Fig. 9(a), the damage spreads widely in the plate and the maximum damage value is the lowest. The opposite result is obtained for the simulation with an isotropic model and the small characteristic length, Fig. 9(b). The damage spreads in a narrow band, and its value at the two edges of the plate, from where the damage is initiated, is much higher than in the central area (around 20\%). However, the simulation with the anisotropic gradient-enhanced model gives a relatively narrow damage band, and the damage distributes more evenly, Fig. 9(c), in the damage band compared to the results obtained of isotropic gradient enhanced model with the small characteristic length, Fig. 9(b). In these simulations, the interactions between elements within a certain distance were taken into account through the gradientenhanced model. With the anisotropic non-local model, the interaction distance between elements can be different in the different material directions, which allows representing with higher accuracy the properties of a transverse isotropic material as in a composite ply.

In the following applications, the following length scales are considered: $c^{3}=2.0 \mathrm{~mm}^{2}$ according to the reference [72] along the fiber directions, and $c^{1}=c^{2}=8.45 \times 10^{-5}$ $\mathrm{mm}^{2}$ in the transverse direction according to the distance between the fibers.

\subsection{Experimental setup}

Specimens of carbon fibers reinforced epoxy composite were manufactured from layers of the prepreg Hexply M10.1/38\%/UD300/HS (R) whose components properties are reported in Section 3.1.

The specimens were cut from an autoclave consolidated unidirectional laminate panel of $300 \times 300 \mathrm{~mm}^{2}$. Two laminates were successively considered: the first one has a $\left[-45_{4}^{\circ} / 45_{4}^{\circ}\right]_{S^{-}}$-stacking sequence and the second one has a $\left[90^{\circ} /+45^{\circ} /-45^{\circ} / 90^{\circ} / 0^{\circ}\right]_{S}$ stacking sequence. The geometries of the samples considered for these two cases are illustrated in Figs. 10(a) and 10(b). To prevent gripping damage, aluminum tabs were glued at both ends of each specimen. For each stacking sequence, between three and five samples coming from a single manufactured plate per laminate, were tested. The reported thickness is an average one, and the discrepancy between samples of a common stacking sequence is up to $0.05 \mathrm{~mm}$. Actual values are however used to extract the strain-stress curves.

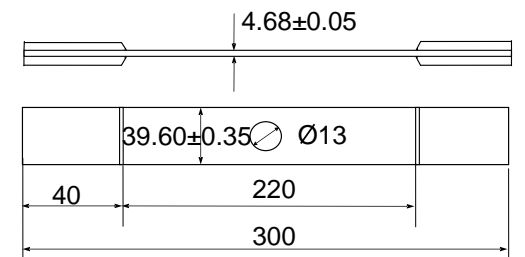

(a) $\left[45_{4}^{o} /-45_{4}^{o}\right]_{S}$

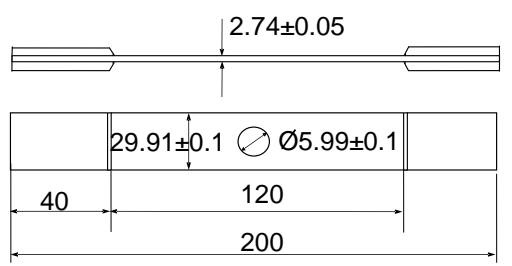

(b) $\left[90^{\circ} /+45^{\circ} /-45^{\circ} / 90^{\circ} / 0^{\circ}\right]_{S}$

Figure 10: Geometry schematics of open-hole experimental specimens (units in $\mathrm{mm}$ ) for (a) the $\left[45_{4}^{\circ} /-45_{4}^{\circ}\right]_{S}$ stacking sequence and (b) the $\left[90^{\circ} /+45^{\circ} /-45^{\circ} / 90^{\circ} / 0^{\circ}\right]_{S}$ stacking sequence.

For the $\left[-45_{4}^{o} / 45_{4}^{o}\right]_{S}$-stacking sequence, static tensile tests were carried out on a $1185 \mathrm{n}^{\circ} \mathrm{H} 4573$ (ME002) Instron machine in displacement control mode with a constant cross-head speed of $2 \mathrm{~mm} / \mathrm{min}$, according to the specification of ISO-527-4 standard. Tensile tests up to failure and up to $90 \%$ of the maximum strength were conducted. The strain-stress curves were extracted from the experimental results by using a $50 \mathrm{~mm}$-long extensometer, as illustrated in Fig. 11.

For the $\left[90^{\circ} /+45^{\circ} /-45^{\circ} / 90^{\circ} / 0^{\circ}\right]_{S}$ stacking sequence, tensile tests were carried out in an electromechanical universal testing 3384 Instron machine in displacement control mode with a constant cross-head speed of $2 \mathrm{~mm} / \mathrm{min}$, according to the specification of ISO-527-4 standard. Tensile tests up to failure and up to $50 \%, 75 \%$, and $90 \%$ of the maximum strength were conducted. The testing device was equipped with a $150 \mathrm{kN}$ load cell. Total strain was measured with the help of an extensometer whose distance between the blades was modified in order to increase as much as possible the region where the strain was registered, reaching $50 \mathrm{~mm}$. X-ray computed tomography (XCT) was performed on selected samples loaded up to $90 \%$ of failure using a Nanotom 160NF (GE Sensing \& Inspection Technologies PhoenixX-ray). The tomograms were collected at $100 \mathrm{kV}$ and $120 \mu \mathrm{A}$ using a tungsten target. For each tomogram, 2,000 radiographs were acquired with an exposure time of $500 \mathrm{~ms}$. The tomogram voxel size was set to $15 \mu \mathrm{m}$. The tomograms were then reconstructed using an algorithm based on the filtered back-projection procedure for Feldkamp cone beam geometry. The damage in the reconstructed volumes was qualitatively and quantitatively analyzed using the freeware ImageJ software and the commercial software VGStudio Max 2.0. Accurate quantification of crack density and delaminated area was possible because of the use of a dye penetrant liquid con- 


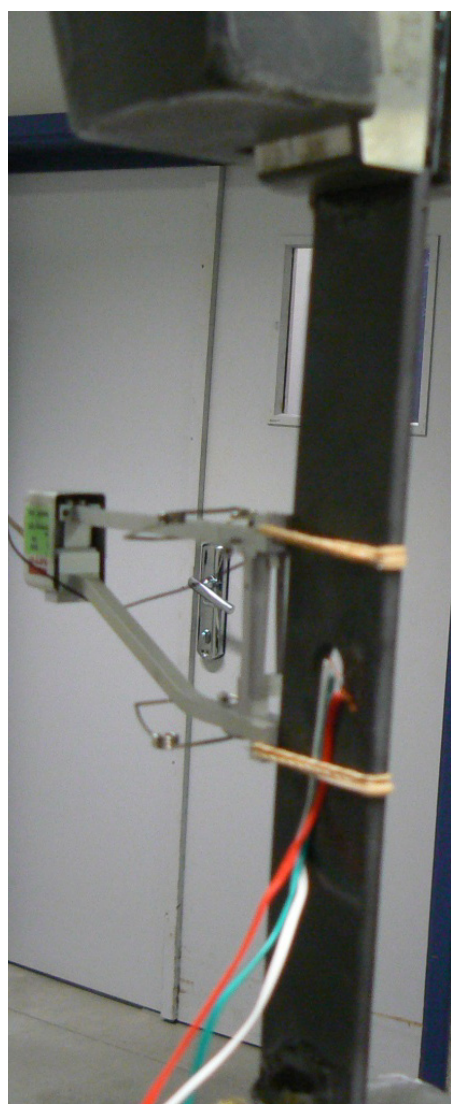

Figure 11: Experimental setup for the tensile tests of the open $\left[45_{4}^{\circ} /-\right.$ $\left.45_{4}^{\circ}\right]_{S}$-hole specimens.

taining $\mathrm{ZnI}$ which caused the cracks and delaminations to appear brighter in the tomograms due to the higher X-ray absorption coefficient of $\mathrm{ZnI}$ as compared with the carbon fibers or the polymeric matrix.

\subsection{Numerical simulation of the open-hole laminates}

In this section, the anisotropic gradient enhanced $\mathrm{MFH}$ model is used to investigate the response of the open hole specimens subjected to uni-axial tension experimentally conducted in Section 3.3.

The numerical models represent the part in-between the extensometer grips, see Figs. 12(a) and 12(c). The geometries are meshed using one bi-quadratic tetrahedron on the thickness of each ply (as the problem is plane stress one element on the thickness was used). Each ply follows a MFH model with the appropriate fibers directions entered as input parameter. The resulting meshes can be seen in Figs. $12(\mathrm{~b})$ and $12(\mathrm{~d})$. For the $\left[90^{\circ} /+45^{\circ} /-45^{\circ} / 90^{\circ} / 0^{\circ}\right]_{S}$ stacking sequence only the upper half of the plate is considered because of the symmetry. For the $\left[-45_{4}^{o} / 45_{4}^{o}\right]_{S}$-stacking sequence, as there are less plies, we consider the full thickness to obtain the final delaminated configuration.

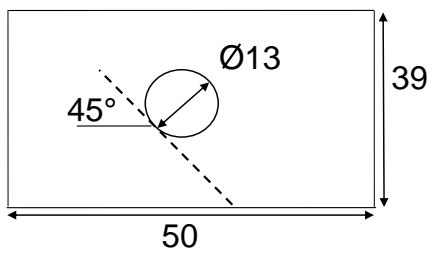

(a) $\left[45_{4}^{\circ} /-45_{4}^{o}\right]_{S}$-model

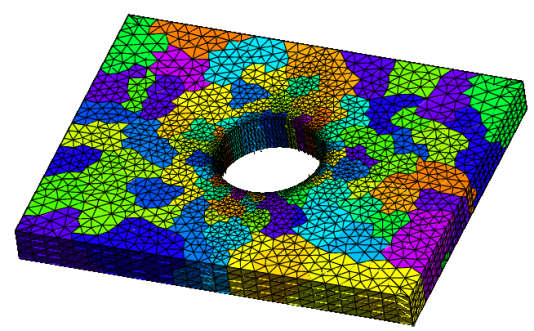

(b) $\left[45_{4}^{o} /-45_{4}^{o}\right]_{S}$-mesh

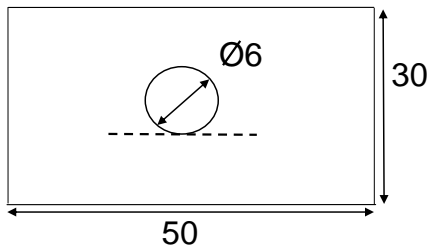

(c) $\left[90^{\circ} /+45^{\circ} /-45^{\circ} / 90^{\circ} / 0^{\circ}\right]_{S^{-}}$ model

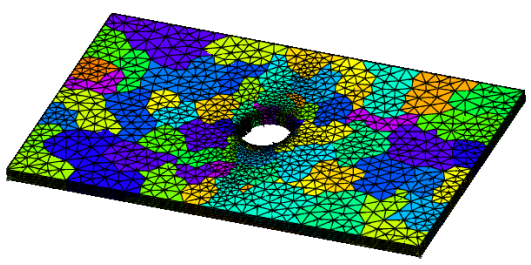

(d) $\left[90^{\circ} /+45^{\circ} /-45^{\circ} / 90^{\circ} / 0^{\circ}\right]_{S}$-mesh

Figure 12: Numerical models. (a) Geometry and (b) finite element mesh for the $\left[45_{4}^{\circ} /-45_{4}^{o}\right]_{S}$ stacking sequence. (c) Geometry and (d) finite element mesh for $\left[90^{\circ} /+45^{\circ} /-45^{\circ} / 90^{\circ} / 0^{\circ}\right]_{S}$ stacking sequence. The different colors in the finite element meshes are related to the different processors of parallel computations.

\subsubsection{Results for the $\left[-45_{4}^{o} / 45_{4}^{o}\right]_{S}$-open hole sample}

Numerical predictions and experimental results are compared in Fig. 13. The stress evolution, evaluated by dividing the tensile load by the plain section, is in good agreement -note the oscillations resulting from the explicit time integration although numerical damping is used and curves are filtered as not every time-steps are representedup to the point of plastic flow. Beyond this state the stress is over-predicted, with a maximum discrepancy near the fracture point, see Fig. 13(a). The reason lies in the imperfection in the experimental plates and in the damaging modes such as debonding, fiber rotations, etc., which are not modeled. Moreover uncertainties remain in the model due to the considered idealized matrix elasto-plastic and 


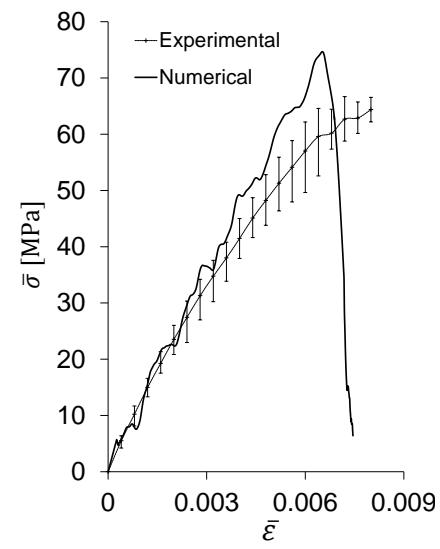

(a) Stress-strain curve

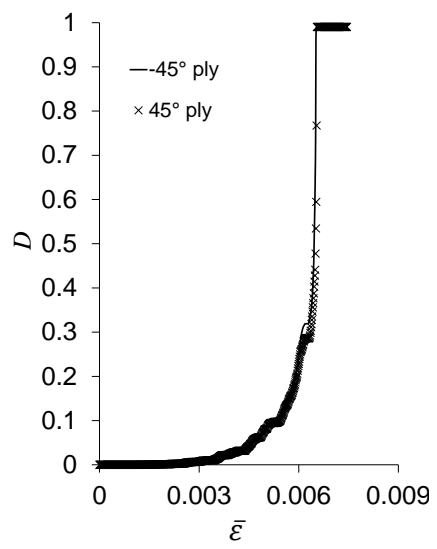

(b) Damage evolution
Figure 13: Tensile test until failure of the $\left[45_{4}^{o} /-45_{4}^{o}\right]_{S}$-open hole sample. (a) Comparison of the apparent stress deduced from the loading force (the experimental strain corresponds to the average strain value measured by a $50 \mathrm{~mm}$-long extensometer). (b) Predicted evolution of the maximum damage reached in each ply.

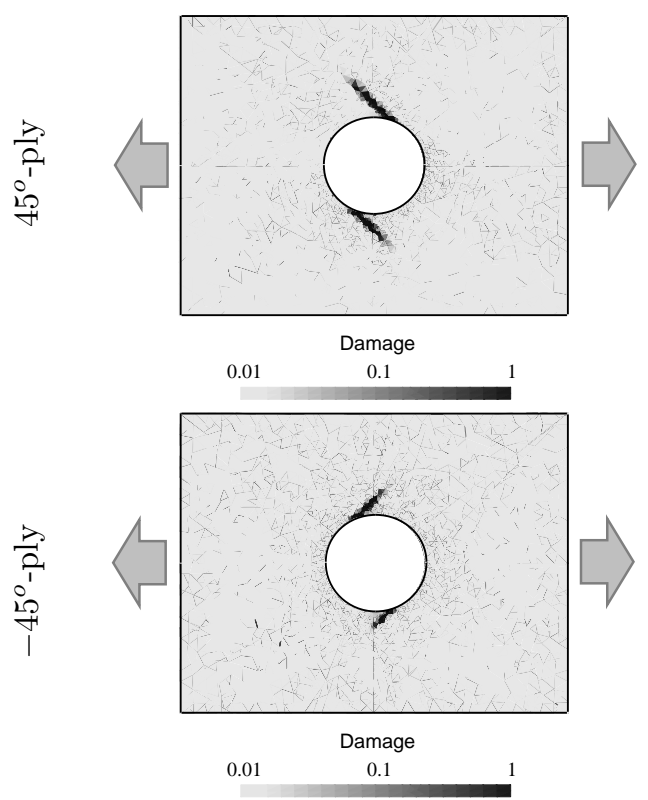

Figure 14: Snapshots of the damage distribution (logarithmic scale) for an average $0.66 \%$ strain in the different plies. The arrows represent the loading direction.

damage evolutions, in the values of some parameters such as the Mode II critical energy release rate, etc. However, the failure stress is captured within $20 \%$ error and the failure strain within $15 \%$ error -which is of the magnitude order of the numerical oscillations.

The evolution of the maximum damage value reached in the matrix phase of each ply is reported in Fig. 13(b). Figure 14 illustrates the damage distributions in the external and internal plies. It can be seen that the maximum damage location of the numerical prediction is in good agreement with the crack initiation locations in the different plies observed for the experimental results, see
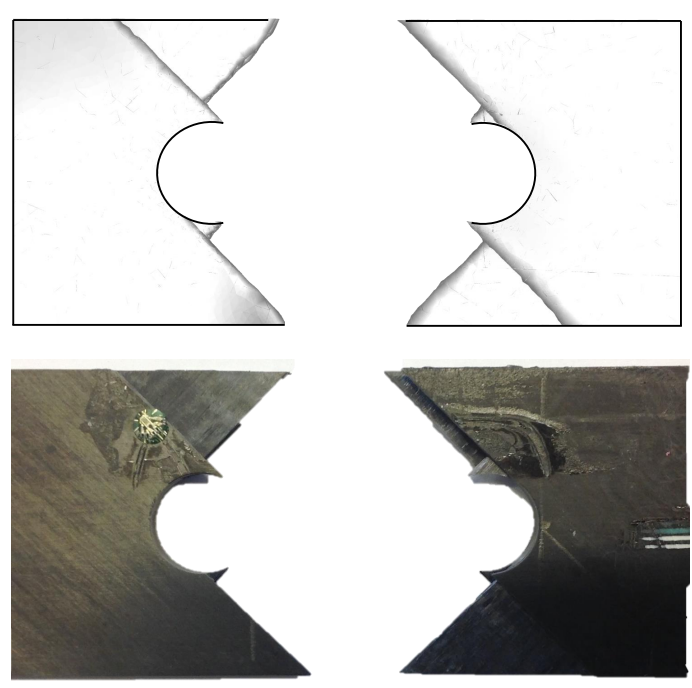

Figure 15: Delaminated parts of the $\left[45_{4}^{\circ} /-45_{4}^{o}\right]_{S}$-open hole sample for the (a) numerical model, (b) experimental sample.

Fig. 15(b). Finally the configuration after total failure is presented for both the numerical and experimental tests in Fig. $15^{3}$. This figure demonstrates the ability of the method to capture the failure mode of the test.

In this application, the damage values are locally beyond the softening point of the matrix behavior, but the damage does not localize in a single element as it would be the case with a local approach. Besides, the non-local approach was shown to be insensitive to the mesh size, thanks to the non-local approach [28].

\subsubsection{Results for the $\left[90^{\circ} /+45^{\circ} /-45^{\circ} / 90^{\circ} / 0^{\circ}\right]_{S}$-open hole sample}

The stress evolution, evaluated by dividing the tensile load by the plain section, obtained with the numerical predictions is compared to the experimental results in Fig. 16(a) and is in good agreement up to the failure point. At this level, as the numerical model does not account for fiber failure, the plies are still sustaining the loading. For the experimental results, three tests were carried out and are found to be almost similar, hence the small size of the error bars. The evolution of the maximum damage value reached in the matrix of each ply is also reported in Fig. 16(b). It can be seen that the damage initiates and propagates first in the $0^{\circ}$-ply, while the outer $90^{\circ}$-ply is the last one to exhibit damage. In all the plies, the damage evolves locally (near the hole as discussed here below) to the maximum value within 0.0002 strain increments.

\footnotetext{
${ }^{3}$ Although strict discontinuities are only modeled at the ply interfaces, the intra-laminar failure model results in finite elements reaching a damage value equal to 1 . As a result, the in-ply displacement field sees a steep slope at the damage bands, which allowed this image of the numerical results to be constructed by successively considering the displacement below and above $0.3 \mathrm{~mm}$ (which is a value above the cohesive model critical openings).
} 

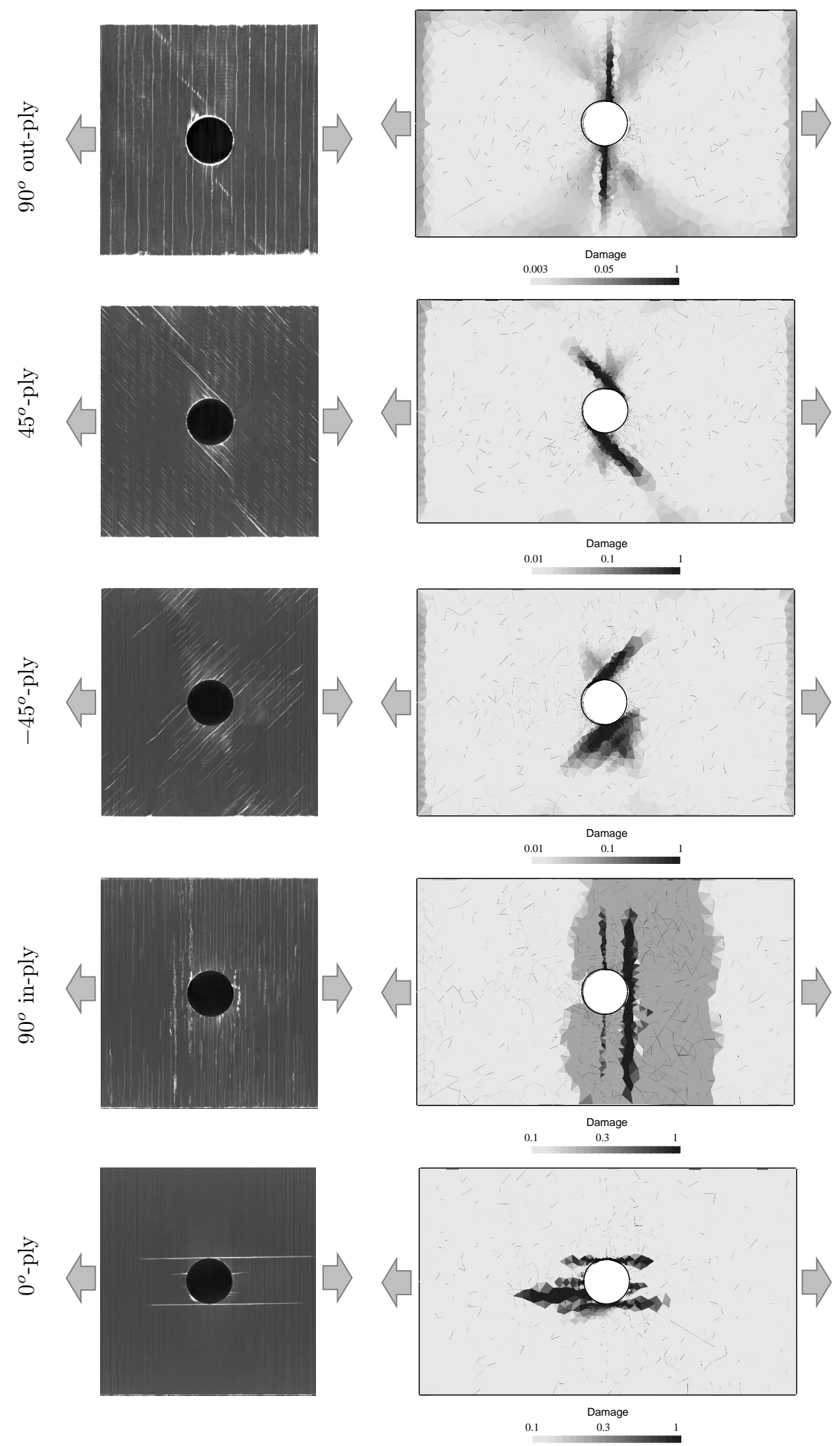

Figure 17: Damage in the different plies of the $\left[90^{\circ} /+45^{\circ} /-45^{\circ} / 90^{\circ} / 0^{\circ}\right]_{S}$-open hole sample at $90 \%$ of the failure. Left column: experimental tomographic results [8]. Right column: numerical prediction (logarithmic scale). The arrows represent the loading direction. 

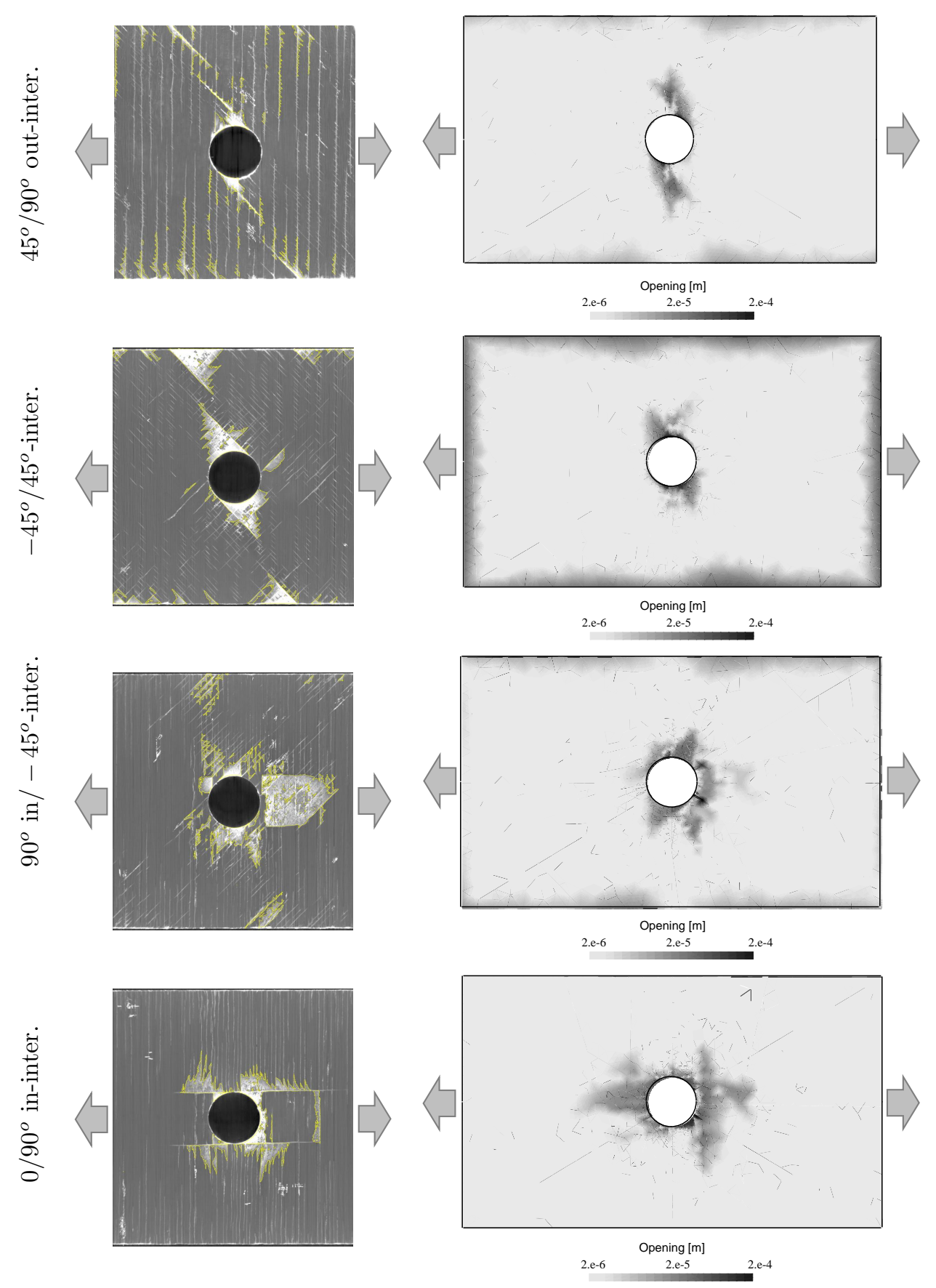

Figure 18: Delaminated parts in the different interfaces of the $\left[90^{\circ} /+45^{\circ} /-45^{\circ} / 90^{\circ} / 0^{\circ}\right]_{S}$-open hole sample at $90 \%$ of the failure. Left column: experimental tomographic results [8]. Right column: numerical predictions (logarithmic scale is saturated at $0.2 \mathrm{~mm}$ ). The arrows represent the loading direction.

The damage distributions in the different plies at $90 \%$ of the failure are reported in Fig. 17 where they are compared to the XCT results. The diffuse damage predicted with the numerical method represents an average value of the damaging process in the matrix of the material while the $\mathrm{XCT}$ is able to capture the micro-crack openings in the material. A direct quantitative comparison is therefore not possible, so the analysis focuses on a qualitative comparison. It can be seen that the maximum damage location of the numerical prediction is in good agreement with the tomographic image:

- In the outer $90^{\circ}$-ply, for both the numerical and experimental results, the damage develops along two directions: along the fiber direction, but also along a $+45^{\circ}$.

- In the $45^{\circ}$-ply, the damage concentrates at the intersection of the hole with a $-45^{\circ}$-line and develops along a $+45^{\circ}$-line. 


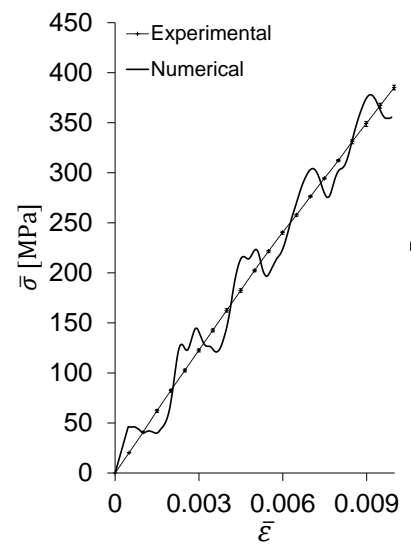

(a) Stress-strain curve

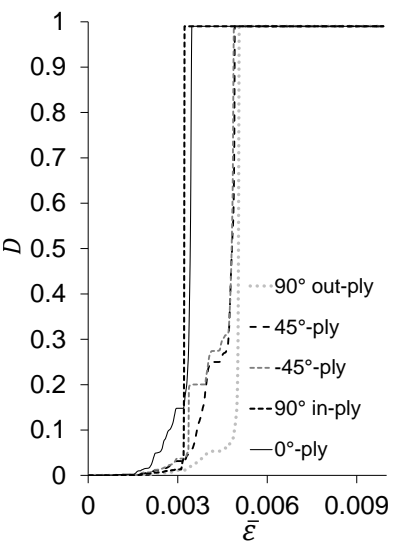

(b) Damage evolution
Figure 16: Tensile test until failure of the $\left[90^{\circ} /+45^{\circ} /-45^{\circ} / 90^{\circ} / 0^{\circ}\right]_{S}$-open hole sample. (a) Comparison of the apparent stress deduced from the loading force for (the experimental strain corresponds to the average strain value measured by a $50 \mathrm{~mm}$-long extensometer). (b) Predicted evolution of the maximum damage reached in each ply.

- In the $-45^{\circ}$-ply, the damage concentrates at the intersection of the hole with a $+45^{\circ}$-line and develops along $\mathrm{a}-45^{\circ}$ line. Both the numerical and experimental results also exhibit damage along the $+45^{\circ}$-line.

- In the central $90^{\circ}$-ply, the damage is more diffuse around the hole, with a preferred-direction along the fibers direction.

- In the central $0^{\circ}$-ply, the damage develops along the fibers direction and initiates tangentially to the hole; The damage extends further with the numerical results at the left side of the hole;

The delaminated parts of the ply interfaces at $90 \%$ of the failure are illustrated in Fig. 18. For the experimental results, the illustration are obtained from superimposed information from the tomographic slices composing the interfaces stacked in the direction perpendicular to the plies. It can be seen that the location and extension of the delaminated zone are in good agreement between the numerical prediction and the experimental observations, and in particular

- At the $45^{\circ} / 90^{\circ}$ out-interface, the delamination is confined in-between these two directions.

- At the $-45^{\circ} / 45^{\circ}$-interface, the delamination takes the typical triangular shape around the hole and delamination at the laminate edges are more important (for the numerical results the delaminated parts at the right and left edges result from the finite length of the mesh);

- At the $90^{\circ}$ in $/-45^{\circ}$-interface, delamination still occurs mainly around the hole at the intersection of the damage zone of the two plies, but there are some parts at the laminate edges as well;

- At the $0 / 90^{\circ}$ in-interface, delamination occurs mainly around the hole at the intersection of the damage zone of the two plies; For the numerical results, the extension of the delaminated zone on the left side of the hole is higher than for the experimental results, due to the higher extension of the damage observed in the $0^{\circ}$ ply, see Fig. 18;

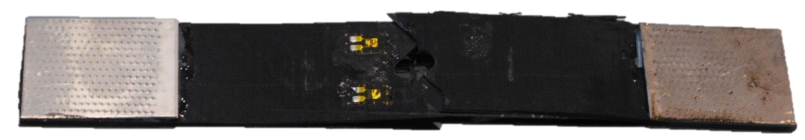

Figure 19: Failure mode of the $\left[90^{\circ} /+45^{\circ} /-45^{\circ} / 90^{\circ} / 0^{\circ}\right]_{S^{-o p e n}}$ hole sample [8].

From this comparison we can conclude that the developed numerical damage-enhanced $\mathrm{MFH}$ scheme coupled with the DG/ECL delamination model is able to predict the damage distribution in complex laminate problems. In particular the damage distribution in the outer-ply, see Fig. 17, is a combination of $+45^{\circ}$ and $90^{\circ}$ evolution, in good agreement with the failure mode observed in Fig. 19.

\section{Conclusions}

In order to analyze the failure of laminated composite structures, an anisotropic gradient-enhanced mean-field homogenization (MFH) procedure has been developed. In this approach, the fibers are assumed to remain linear elastic while the matrix material obeys an elasto-plastic behavior enhanced by a damage model. The loss of solution uniqueness at onset of strain softening has been avoided by using a non-local implicit approach, reformulated in an anisotropic way, to describe the damage in the matrix.

To capture the failure mode of the composite laminates, delamination is also modeled. To this end, the hybrid DG/ECL model integrates the traction separation law at the interface between plies. On the one hand this hybrid method avoids the need to propagate topological changes in the mesh with the propagation of the delamination, and on the other hand it preserves the consistency and stability in the un-cracked interfaces, thus avoiding the change of compliance issues common to the use of intrinsic cohesive models.

As a demonstration of the method abilities, two openhole laminates with different stacking sequences are studied. It is shown that the model predicts within each ply the damaging process in bands oriented with the experimentally observed failure mode. Moreover the regions of high damage values in the different ply are in qualitative agreement with the crack initiation locations observed in the experiments.

In the future a more evolved material model for the matrix material, including the damage law, will be considered 
and characterized directly with experiments conducted on the epoxy. Damage-to-crack transition scheme will also be developed for intra-laminar failure in order to model the fracture observed during the experiments.

\section{Acknowledgment}

The authors acknowledge funding through SIMUCOMP, an ERA-NET +, MATERA+ project financed by Consejería de Educación y Empleo of Comunidad de Madrid, the Walloon region (agreement $n^{o}$ 1017232, CT-EUC 201010-12), the Luxembourg region, Fonds National de la Recherche de Luxembourg, and by the European Unionś Seventh Framework Programme (FP7/2007-2013).

Computational resources have been provided by the supercomputing facilities of the Consortium des Équipements de Calcul Intensif en Fédération Wallonie Bruxelles (CÉCI) funded by the Fond de la Recherche Scientifique de Belgique (FRS-FNRS).

\section{References}

[1] P. Ladevèze, G. Lubineau, D. Marsal, Towards a bridge between the micro- and mesomechanics of delamination for laminated composites, Composites Science and Technology 66 (6) (2006) 698 - 712, ISSN 0266-3538, doi: 10.1016/j.compscitech.2004.12.026.

[2] P. Maimí, P. Camanho, J. Mayugo, C. Dávila, A continuum damage model for composite laminates: Part I-Constitutive model, Mechanics of Materials 39 (10) (2007) 897-908.

[3] P. Maimí, P. Camanho, J. Mayugo, C. Dávila, A continuum damage model for composite laminates: Part II Computational implementation and validation, Mechanics of Materials 39 (10) (2007) 909 - 919, ISSN 0167-6636.

[4] J. LLorca, C. González, J. M. Molina-Aldareguía, J. Segurado, R. Seltzer, F. Sket, M. Rodríguez, S. Sádaba, R. Muñoz, L. P. Canal, Multiscale Modeling of Composite Materials: a Roadmap Towards Virtual Testing, Advanced Materials 23 (44) (2011) 5130-5147, ISSN 1521-4095, doi: 10.1002/adma.201101683.

[5] S. Pinho, L. Iannucci, P. Robinson, Physically based failure models and criteria for laminated fibre-reinforced composites with emphasis on fibre kinking. Part II: FE implementation, Composites Part A: Applied Science and Manufacturing 37 (5) (2006) 766 - 777, ISSN 1359-835X.

[6] P. Camanho, P. Maimí, C. Dávila, Prediction of size effects in notched laminates using continuum damage mechanics, Composites Science and Technology 67 (13) (2007) 2715 - 2727, ISSN 0266-3538, doi:10.1016/j.compscitech.2007.02.005.

[7] O. Allix, P. Feissel, P. Thévenet, A delay damage mesomodel of laminates under dynamic loading: basic aspects and identification issues, Computers \& Structures 81 (12) (2003) 1177 - 1191, ISSN 0045-7949, doi:10.1016/S0045-7949(03)00035-X, advanced Computational Models and Techniques in Dynamics.

[8] G. Vigueras, F. Sket, C. Samaniego, L. Wu, L. Noels, D. Tjahjanto, E. Casoni, G. Houzeaux, A. Makradi, J. M. MolinaAldareguia, M. Vazquez, A. Jérusalem, An XFEM/CZM implementation for massively parallel simulations of composites fracture, Composite Structures - (0) (In Press) -, doi: 10.1016/j.compstruct.2015.01.053.

[9] F. van der Meer, L. Sluys, A phantom node formulation with mixed mode cohesive law for splitting in laminates, International Journal of Fracture 158 (2) (2009) 107-124, ISSN 03769429, doi:10.1007/s10704-009-9344-5.
[10] P. Kanouté, D. Boso, J. Chaboche, B. Schrefler, Multiscale Methods for Composites: A Review, Archives of Computational Methods in Engineering 16 (2009) 31-75, ISSN 1134-3060, 10.1007/s11831-008-9028-8.

[11] M. Geers, V. Kouznetsova, W. Brekelmans, Multi-scale computational homogenization: Trends and challenges, Journal of Computational and Applied Mathematics 234 (7) (2010) 2175 - 2182, ISSN 0377-0427, doi:10.1016/j.cam.2009.08.077.

[12] I. Doghri, L. Brassart, L. Adam, J. S. Gérard, A secondmoment incremental formulation for the mean-field homogenization of elasto-plastic composites, International Journal of Plasticity 27 (3) (2011) 352 - 371, ISSN 0749-6419, doi: 10.1016/j.ijplas.2010.06.004.

[13] J. D. Eshelby, The Determination of the Elastic Field of an Ellipsoidal Inclusion, and Related Problems, Proceedings of the Royal Society of London. Series A, Mathematical and Physical Sciences 241 (1226) (1957) pp. 376-396, ISSN 00804630.

[14] T. Mori, K. Tanaka, Average stress in matrix and average elastic energy of materials with misfitting inclusions, Acta Metallurgica 21 (5) (1973) 571-574, cited By (since 1996) 1814.

[15] Y. Benveniste, A new approach to the application of MoriTanaka's theory in composite materials, Mechanics of Materials 6 (2) (1987) 147 - 157, ISSN 0167-6636, doi:10.1016/01676636(87)90005-6.

[16] E. Kröner, Berechnung der elastischen Konstanten des Vielkristalls aus den Konstanten des Einkristalls, Zeitschrift für Physik A Hadrons and Nuclei 151 (1958) 504-518, ISSN 09397922, 10.1007/BF01337948.

[17] R. Hill, A self-consistent mechanics of composite materials, Journal of the Mechanics and Physics of Solids 13 (4) (1965) 213 - 222, ISSN 0022-5096, doi:10.1016/0022-5096(65)90010-4.

[18] H. E. Pettermann, A. F. Plankensteiner, H. J. Böhm, F. G. Rammerstorfer, A thermo-elasto-plastic constitutive law for inhomogeneous materials based on an incremental Mori-Tanaka approach, Computers \& Structures 71 (2) (1999) $197-214$, ISSN 0045-7949, doi:10.1016/S0045-7949(98)00208-9.

[19] I. Doghri, A. Ouaar, Homogenization of two-phase elasto-plastic composite materials and structures: Study of tangent operators, cyclic plasticity and numerical algorithms, International Journal of Solids and Structures 40 (7) (2003) 1681 - 1712, ISSN 00207683, doi:10.1016/S0020-7683(03)00013-1.

[20] J. Chaboche, P. Kanouté, A. Roos, On the capabilities of meanfield approaches for the description of plasticity in metal matrix composites, International Journal of Plasticity 21 (7) (2005) 1409 - 1434, ISSN 0749-6419, doi:10.1016/j.ijplas.2004.07.001.

[21] I. Doghri, L. Tinel, Micromechanical modeling and computation of elasto-plastic materials reinforced with distributed-orientation fibers, International Journal of Plasticity 21 (10) (2005) 1919 - 1940, ISSN 0749-6419, doi:10.1016/j.ijplas.2004.09.003.

[22] O. Pierard, I. Doghri, Study of Various Estimates of the Macroscopic Tangent Operator in the Incremental Homogenization of Elastoplastic Composites, International Journal for Multiscale Computational Engineering 4 (4) (2006) 521-543, ISSN 15431649 .

[23] O. Pierard, J. LLorca, J. Segurado, I. Doghri, Micromechanics of particle-reinforced elasto-viscoplastic composites: Finite element simulations versus affine homogenization, International Journal of Plasticity 23 (6) (2007) 1041 - 1060, ISSN 0749-6419, doi:10.1016/j.ijplas.2006.09.003.

[24] I. Doghri, L. Adam, N. Bilger, Mean-field homogenization of elasto-viscoplastic composites based on a general incrementally affine linearization method, International Journal of Plasticity 26 (2) (2010) 219 - 238, ISSN 0749-6419, doi: 10.1016/j.ijplas.2009.06.003.

[25] S. Mercier, A. Molinari, Homogenization of elasticviscoplastic heterogeneous materials: Self-consistent and Mori-Tanaka schemes, International Journal of Plasticity 25 (6) (2009) 1024 - 1048, ISSN 0749-6419, doi:10.1016/j.ijplas.2008.08.006.

[26] L. Wu, L. Noels, L. Adam, I. Doghri, A combined incrementalsecant mean-field homogenization scheme with per-phase resid- 
ual strains for elasto-plastic composites, International Journal of Plasticity 51 (2013) 80-102, doi:10.1016/j.ijplas.2013.06.006.

27] L. Wu, L. Noels, L. Adam, I. Doghri, A multiscale mean-field homogenization method for fiber-reinforced composites with gradient-enhanced damage models, Computer Methods in Applied Mechanics and Engineering 233-236 (2012) 164-179, doi: 10.1016/j.cma.2012.04.011.

[28] L. Wu, L. Noels, L. Adam, I. Doghri, An implicit-gradientenhanced incremental-secant mean-field homogenization scheme for elasto-plastic composites with damage, International Journal of Solids and Structures 50 (24) (2013) 3843 - 3860, ISSN 0020-7683, doi:10.1016/j.ijsolstr.2013.07.022.

[29] R. Peerlings, R. de Borst, W. Brekelmans, S. Ayyapureddi, Gradient-enhanced damage for quasi-brittle materials, Int. J. Numer. Meth. Engng 39 (1996) 3391-3403.

[30] M. Geers, Experimental Analysis and Computational Modelling of Damage and Fracture, Ph.D. thesis, University of Technology, Eindhoven (Netherlands)., 1997.

[31] R. Peerlings, R. de Borst, W. Brekelmans, M. Geers, Gradientenhanced damage modelling of concrete fracture, Mech. Cohesive-Frictional Mat. 3 (1998) 323-342.

[32] R. Peerlings, M. Geers, R. de Borst, W. Brekelmans, A critical comparison of nonlocal and gradient-enhanced softening continua, Int. J. Solids Structures 38 (2001) 7723-7746.

[33] P. P. Camanho, C. G. Dãvila, M. F. de Moura, Numerical Simulation of Mixed-Mode Progressive Delamination in Composite Materials, Journal of Composite Materials 37 (16) (2003) 14151438, doi:10.1177/0021998303034505.

[34] P. W. Harper, S. R. Hallett, Cohesive zone length in numerical simulations of composite delamination, Engineering Fracture Mechanics 75 (16) (2008) 4774 - 4792, ISSN 0013-7944, doi:10.1016/j.engfracmech.2008.06.004.

[35] W.-G. Jiang, S. R. Hallett, B. G. Green, M. R. Wisnom, A concise interface constitutive law for analysis of delamination and splitting in composite materials and its application to scaled notched tensile specimens, International Journal for Numerical Methods in Engineering 69 (9) (2007) 1982-1995, ISSN 10970207, doi:10.1002/nme.1842.

[36] S. Pinho, L. Iannucci, P. Robinson, Formulation and implementation of decohesion elements in an explicit finite element code, Composites Part A: Applied Science and Manufacturing 37 (5) (2006) 778 - 789, doi:10.1016/j.compositesa.2005.06.007.

[37] A. Turon, P. Camanho, J. Costa, C. Dãvila, A damage model for the simulation of delamination in advanced composites under variable-mode loading, Mechanics of Materials 38 (11) (2006) 1072 - 1089, ISSN 0167-6636, doi: 10.1016/j.mechmat.2005.10.003.

[38] A. Turon, C. Dãvila, P. Camanho, J. Costa, An engineering solution for mesh size effects in the simulation of delamination using cohesive zone models, Engineering Fracture Mechanics 74 (10) (2007) 1665 - 1682, ISSN 0013-7944, doi: 10.1016/j.engfracmech.2006.08.025.

[39] G. T. Camacho, M. Ortiz, Computational modelling of impact damage in brittle materials, International Journal of Solids and Structures 33 (20-22) (1996) 2899-2938, ISSN 0020-7683, doi: 10.1016/0020-7683(95)00255-3.

[40] A. Pandolfi, P. Guduru, M. Ortiz, A. Rosakis, Three dimensional cohesive-element analysis and experiments of dynamic fracture in C300 steel, International Journal of Solids and Structures 37 (27) (2000) 3733 - 3760, ISSN 0020-7683, doi: 10.1016/S0020-7683(99)00155-9.

[41] J. Mergheim, E. Kuhl, P. Steinmann, A hybrid discontinuous Galerkin/interface method for the computational modelling of failure, Communications in Numerical Methods in Engineering 20 (7) (2004) 511-519.

[42] R. Radovitzky, A. Seagraves, M. Tupek, L. Noels, A scalable 3D fracture and fragmentation algorithm based on a hybrid, discontinuous Galerkin, cohesive element method, Computer Methods in Applied Mechanics and Engineering 200 (2011) 326-344, ISSN 0045-7825, doi:10.1016/j.cma.2010.08.014.

[43] M. Prechtel, G. Leugering, P. Steinmann, M. Stingl, To- wards optimization of crack resistance of composite materials by adjustment of fiber shapes, Engineering Fracture Mechanics 78 (6) (2011) 944 - 960, ISSN 0013-7944, doi: 10.1016/j.engfracmech.2011.01.007.

[44] L. Wu, D. Tjahjanto, G. Becker, A. Makradi, A. Jérusalem, L. Noels, A micromeso-model of intra-laminar fracture in fiber-reinforced composites based on a discontinuous Galerkin/cohesive zone method, Engineering Fracture Mechanics 104 (2013) 162-183, ISSN 0013-7944, doi: 10.1016/j.engfracmech.2013.03.018.

[45] G. Becker, L. Noels, A full discontinuous Galerkin formulation of non-linear Kirchhoff-Love shells: elasto-plastic finite deformations, parallel computation \& fracture applications, International Journal for Numerical Methods in Engineering 93 (2013) 80-117, ISSN 1097-0207, doi:10.1002/nme.4381.

[46] Y. Charles, A finite element formulation to model extrinsic interfacial behavior, Finite Elements in Analysis and Design 88 (0) (2014) 55-66, ISSN 0168-874X, doi:10.1016/j.finel.2014.05.008.

[47] V. P. Nguyen, An open source program to generate zerothickness cohesive interface elements, Advances in Engineering Software 74 (0) (2014) 27 - 39, ISSN 0965-9978, doi: 10.1016/j.advengsoft.2014.04.002.

[48] V. P. Nguyen, Discontinuous Galerkin/extrinsic cohesive zone modeling: Implementation caveats and applications in computational fracture mechanics, Engineering Fracture Mechanics 128 (2014) 37-68, ISSN 0013-7944, doi: 10.1016/j.engfracmech.2014.07.003.

[49] M. Geers, R. de Borst, W. Brekelmans, R. Peerlings, Validation and internal length scale determination for a gradient damage model: application to short glass-fibre-reinforced polypropylene, International Journal of Solids and Structures 36 (17) (1999) 2557 - 2583, ISSN 0020-7683.

50] L. Wu, L. Noels, L. Adam, I. Doghri, Non-local DamageEnhanced MFH for Multiscale Simulations of Composites, in: Composite Materials and Joining Technologies for Composites, Volume 7, chap. 13, Springer, doi:10.1007/978-1-4614-45531_13, 2013.

[51] F. van der Meer, L. Sluys, Continuum Models for the Analysis of Progressive Failure in Composite Laminates, Journal of Composite Materials 43 (20) (2009) 2131-2156, doi: $10.1177 / 0021998309343054$.

52] E. Abisset, F. Daghia, P. Ladevèze, On the validation of a damage mesomodel for laminated composites by means of open-hole tensile tests on quasi-isotropic laminates, Composites Part A: Applied Science and Manufacturing 42 (10) (2011) 1515 - 1524, ISSN 1359-835X, doi:10.1016/j.compositesa.2011.07.004.

[53] F. P. van der Meer, L. J. Sluys, S. R. Hallett, M. R. Wisnom, Computational modeling of complex failure mechanisms in laminates, Journal of Composite Materials 46 (5) (2012) 603-623, doi: $10.1177 / 0021998311410473$.

[54] B. Chen, T. Tay, P. Baiz, S. Pinho, Numerical analysis of size effects on open-hole tensile composite laminates, Composites Part A: Applied Science and Manufacturing 47 (0) (2013) 52 62, ISSN 1359-835X, doi:10.1016/j.compositesa.2012.12.001.

[55] Z. P. Bažant, B. Oh, Crack band theory for fracture of concrete, Matériaux et Construction 16 (1983) 155-177, ISSN 0025-5432, doi:10.1007/BF02486267.

[56] R. De Borst, Simulation of strain localization: a reappraisal of the Cosserat continuum, Engineering Computations 8 (4) (1991) 317 - 332, ISSN 0264-4401.

[57] Z. P. Bažant, T. B. Belytchko, T. P. Chang, Continuum Theory for Strain-Softening, Journal of Engineering MechanicsASCE 110 (12) (1984) 1666-1692, doi:10.1061/(ASCE)07339399(1984)110:12(1666).

[58] H. M. Zbib, E. C. Aifantis, A Gradient-Dependent Flow Theory of Plasticity: Application to Metal and Soil Instabilities, Applied Mechanics Reviews 42 (11S) (1989) S295-S304, doi: 10.1115/1.3152403.

[59] E. Lorentz, S. Andrieux, A variational formulation for nonlocal damage models, International Journal of Plasticity 15 (2) (1999) 119 - 138, ISSN 0749-6419, doi:10.1016/S0749-6419(98)00057-6. 
[60] E. Aifantis, On the role of gradients in the localization of deformation and fracture, International Journal of Engineering Science 30 (10) (1992) 1279 - 1299, ISSN 0020-7225, doi: 10.1016/0020-7225(92)90141-3.

[61] T. Svedberg, K. Runesson, A thermodynamically consistent theory of gradient-regularized plasticity coupled to damage, International Journal of Plasticity 13 (67) (1997) 669 - 696, ISSN 0749-6419, doi:10.1016/S0749-6419(97)00033-8.

[62] R. Peerlings, T. Massart, M. Geers, A thermodynamically motivated implicit gradient damage framework and its application to brick masonry cracking, Computer Methods in Applied Mechanics and Engineering 193 (30) (2004) 3403 - 3417, ISSN 0045-7825, doi:10.1016/j.cma.2003.10.021.

[63] L. Noels, R. Radovitzky, A general discontinuous Galerkin method for finite hyperelasticity. Formulation and numerical applications, International Journal for Numerical Methods in Engineering 68 (1) (2006) 64-97, doi:10.1002/nme.1699.

[64] L. Wu, G. Becker, L. Noels, Elastic damage to crack transition in a coupled non-local implicit discontinuous Galerkin/extrinsic cohesive law framework, Computer Methods in Applied Mechanics and Engineering 279 (2014) 379-409, ISSN 0045-7825, doi:10.1016/j.cma.2014.06.031.

[65] G. M. Hulbert, J. Chung, Explicit time integration algorithms for structural dynamics with optimal numerical dissipation, Computer Methods in Applied Mechanics and Engineering 137 (2) (1996) 175 - 188, ISSN 0045-7825, doi:10.1016/S00457825(96)01036-5.

[66] J. Mediavilla, R. Peerlings, M. Geers, An integrated continuousdiscontinuous approach towards damage engineering in sheet metal forming processes, Engineering Fracture Mechanics 73 (7) (2006) 895-916.

[67] C. Geuzaine, J.-F. Remacle, Gmsh: A 3-D finite element mesh generator with built-in pre- and post-processing facilities, International Journal for Numerical Methods in Engineering 79 (11) (2009) 1309-1331, doi:10.1002/nme.2579.

[68] J. Lemaitre, Coupled elasto-plasticity and damage constitutive equations, Computer Methods in Applied Mechanics and Engineering 51 (1-3) (1985) 31 - 49, ISSN 0045-7825, doi: 10.1016/0045-7825(85)90026-X

[69] J. Lemaitre, J.-L. Chaboche, Mécanique des Matériaux solides, Dunod, ISBN 2040157867, 1991.

[70] I. Doghri, Numerical implementation and analysis of a class of metal plasticity models coupled with ductile damage, International Journal for Numerical Methods in Engineering 38 (20) (1995) 3403-3431, ISSN 1097-0207, doi: $10.1002 /$ nme. 1620382004 .

[71] J. Byström, Optimal design of a long and slender compressive strut, The International Journal of Multiphysics 3 (2009) 235257, doi:10.1260/175095409788922275.

[72] M. G. D. Geers, R. de Borst, W. A. M. Brekelmans, R. H. J. Peerlings, Strain-based transient-gradient damage model for failure analyses, Computer methods in applied mechanics and engineering 160 (1998) 133-153.

[73] L. Noels, R. Radovitzky, An explicit discontinuous Galerkin method for non-linear solid dynamics: Formulation, parallel implementation and scalability properties, International Journal for Numerical Methods in Engineering 74 (9) (2008) 1393-1420, ISSN 1097-0207, doi:10.1002/nme.2213.

\section{Appendix A. Anisotropic implicit gradient for- mulation}

Following the same process as for isotropic materials [32], an alternative gradient formulation can be derived in the local coordinates. For an anisotropic material, we define the characteristic volume $\left(V_{C}\right)$ in the local coordinates $\left(l_{1}, l_{2}, l_{3}\right)$. For simplicity, we fix the origin of the coordinates at the material point under consideration.
The non-local equation (1) can be rewritten in the local coordinates as

$$
\tilde{a}(\mathbf{0})=\frac{1}{V_{C}} \int_{V_{C}} a(\boldsymbol{l}) \phi(\boldsymbol{l} ; \mathbf{0}) \mathrm{d} V,
$$

where $\boldsymbol{l}$ denotes the position of the infinitesimal volume $\mathrm{d} V_{C}$ in terms of the local coordinates. Thus, a Taylor expansion for $a$ in these local axes reads

$$
\begin{aligned}
a(\boldsymbol{l})= & a(\mathbf{0})+\left.\frac{\partial a}{\partial l_{i}}\right|_{\mathbf{0}} l_{i}+\left.\frac{1}{2 !} \frac{\partial^{2} a}{\partial l_{i} \partial l_{j}}\right|_{\mathbf{0}} l_{i} l_{j}+ \\
& \left.\frac{1}{3 !} \frac{\partial^{3} a}{\partial l_{i} \partial l_{j} \partial l_{k}}\right|_{\mathbf{0}} l_{i} l_{j} l_{k}+ \\
& \left.\frac{1}{4 !} \frac{\partial^{4} a}{\partial l_{i} \partial l_{j} \partial l_{k} \partial l_{l}}\right|_{\mathbf{0}} l_{i} l_{j} l_{k} l_{l}+\cdots .
\end{aligned}
$$

Substituting this relation into (A.1) and integrating on the characteristic volume $\left(V_{C}\right)$ yield

$$
\tilde{a}(\mathbf{0})=a(\mathbf{0})+\left.c_{2}^{i} \frac{\partial^{2} a}{\partial l_{i}{ }^{2}}\right|_{\mathbf{0}}+\left.c_{4}^{i j} \frac{\partial^{4} a}{\partial l_{i}{ }^{2} \partial l_{j}{ }^{2}}\right|_{\mathbf{0}}+\cdots,
$$

where $c_{2}^{i}$ and $c_{4}^{i j}(i, j=1,2,3)$ are coefficients obtained from the integration, and related to the dimensions of the characteristic volume $V_{C}$. Note that odd orders and cross derivatives annihilate due to the symmetry of the weight functions and of the characteristic volume.

Let us compute $c_{2}^{i} \frac{\partial^{2} \tilde{a}}{\partial l_{i}{ }^{2}}$ from (A.3), yielding

$$
c_{2}^{i} \frac{\partial^{2} \tilde{a}}{\partial l_{i}{ }^{2}}=c_{2}^{i} \frac{\partial^{2} a}{\partial l_{i}{ }^{2}}+\left(2-\delta_{i j}\right) c_{2}^{i} c_{2}^{j} \frac{\partial^{4} a}{\partial l_{i}{ }^{2} \partial l_{j}{ }^{2}}+\cdots,
$$

where $\delta_{i j}$ is Kronecker's symbol. In this expression, we omit the origin "0" without losing its generality. Subtracting this relation from (A.3) allows writing

$$
\tilde{a}-c_{2} \frac{\partial^{2} \tilde{a}}{\partial l_{i}{ }^{2}}=a+\left[c_{4}^{i j}-\left(2-\delta_{i j}\right) c_{2}^{i} c_{2}^{j}\right] \frac{\partial^{4} a}{\partial l_{i}{ }^{2} \partial l_{j}{ }^{2}}+\cdots .
$$

Neglecting terms of order four and higher in the right-hand side, another approximation of the non-local form (A.1) is obtained:

$$
\tilde{a}-c_{2}^{i} \frac{\partial^{2} \tilde{a}}{\partial l_{i}{ }^{2}}=a .
$$

Replacing $c_{2}^{i}$ by $c^{i}$ without possible confusion, and using the definition of the characteristic length tensor $\boldsymbol{c}_{1}=$ $\operatorname{diag}\left(c^{i}\right)$, the equation (A.6) is rewritten as

$$
\tilde{a}-\nabla_{l} \cdot\left(c_{1} \cdot \nabla_{l} \tilde{a}\right)=a,
$$

where " $\nabla_{l}$ " and " $\nabla_{l}$ " " represent the gradient operator and the divergence operator expressed in the local coordinates, respectively. In this expression, each term $c^{i}$ of the diagonal tensor $c_{1}$ represents the square of the characteristic length along the direction $l_{i}$.

In order to get the unique solution of this differential equation, a boundary condition must be provided. The following boundary condition is used:

$$
\left(\boldsymbol{c}_{1} \cdot \nabla_{l} \tilde{a}\right) \cdot \boldsymbol{n}_{\mathrm{l}}=0,
$$


with $\boldsymbol{n}_{1}$ the unit normal to $\Gamma$, the boundary of the entire problem domain $\Omega$, expressed in the local coordinates. Then, in the entire problem domain $\Omega$, one has

$$
\begin{aligned}
& \int_{\Omega}\left[\tilde{a}-\nabla_{l} \cdot\left(\boldsymbol{c}_{\mathrm{l}} \cdot \boldsymbol{\nabla}_{l} \tilde{a}\right)\right] \mathrm{d} \Omega= \\
& \int_{\Omega} \tilde{a} \mathrm{~d} \Omega-\int_{\Omega} \nabla_{l} \cdot\left(\boldsymbol{c}_{\mathrm{l}} \cdot \boldsymbol{\nabla}_{l} \tilde{a}\right) \mathrm{d} \Omega= \\
& \int_{\Omega} \tilde{a} \mathrm{~d} \Omega-\oint_{\Gamma}\left(\boldsymbol{c}_{\mathrm{l}} \cdot \boldsymbol{\nabla}_{l} \tilde{a}\right) \cdot \boldsymbol{n}_{\mathrm{l}} \mathrm{d} \Gamma .
\end{aligned}
$$

According to governing equation (A.7) and to the presented boundary condition (A.8), we have

$$
\int_{\Omega} \tilde{a} \mathrm{~d} \Omega=\int_{\Omega} a \mathrm{~d} \Omega .
$$

This last relation demonstrates that the non-local formulation respects the average values on the entire domain.

As the material is not always oriented accordingly to the global coordinates, the gradient enhanced formulation should be stated in the inertial frame. A rotation tensor $\boldsymbol{R}$ is defined to represent the change of orthonormal coordinates from global to local. This rotation tensor $\boldsymbol{R}$ can be formulated from the Euler angles as

$$
\boldsymbol{R}=\left[\begin{array}{ccc}
c_{1} c_{3}-c_{2} s_{1} s_{3} & c_{1} c_{2} s_{3}+c_{3} s_{1} & s_{2} s_{3} \\
-c_{1} s_{3}-s_{1} c_{2} c_{3} & c_{1} c_{2} c_{3}-s_{1} s_{3} & c_{3} s_{2} \\
s_{1} s_{2} & -c_{1} s_{2} & c_{2}
\end{array}\right]
$$

where $c_{k}=\cos \left(\theta_{k}\right), s_{k}=\sin \left(\theta_{k}\right),(k=1,2,3)$, and where $\theta_{k}$ is the $k^{\text {th }}$ Euler angle.

The characteristic length tensor is thus defined in the global coordinates as

$$
\boldsymbol{c}_{\mathrm{g}}=\boldsymbol{R}^{T} \cdot \boldsymbol{c}_{\mathrm{l}} \cdot \boldsymbol{R}
$$

As $\frac{\partial X_{i}}{\partial l_{j}}=R_{j i}=R_{i j}^{T}(i, j=1,, 2,3)$, the anisotropic implicit gradient formulation stated in the global coordinates (A.7) is rewritten

$$
\tilde{a}-\nabla \cdot\left(c_{\mathrm{g}} \cdot \nabla \tilde{a}\right)=a,
$$

and is completed by the boundary condition (A.8), which becomes in the global coordinates

$$
\left(\boldsymbol{c}_{\mathrm{g}} \cdot \nabla \tilde{a}\right) \cdot \boldsymbol{n}=0 .
$$

In these last equations " $\nabla$ " and " $\nabla$ " represent the gradient operator and the divergence operator in global coordinates, respectively, and $\boldsymbol{n}$ is the outward unit normal expressed in the global coordinates.

\section{Appendix B. Expressions of the finite element forces}

The elementary finite-element forces can be computed by inserting the polynomial approximations (22-23) into the weak form (20-21), which leads directly to the following expressions of the elementary (bulk elements $\Omega^{e}$ and interface elements $\Gamma_{\mathrm{L}}^{s}$ ) nodal forces

$$
\begin{aligned}
& \boldsymbol{f}_{\overline{\boldsymbol{u}} \text { int }}^{e}{ }^{m}=\int_{\Omega^{e}} \boldsymbol{\sigma} \cdot \nabla N^{m} d V \\
& \boldsymbol{f}_{\overline{\boldsymbol{u}} \text { ext }}^{e}{ }^{a}=\int_{\Omega^{e}} \overline{\boldsymbol{\rho}} \overline{\boldsymbol{b}} N^{m} d V+\int_{\Gamma_{\mathrm{N}}} N^{m} \cdot \overline{\overline{\boldsymbol{t}}} d s \\
& \boldsymbol{f}_{\overline{\boldsymbol{u}} \mathrm{L}}^{s} m_{i}^{i+1}= \pm \int_{\Gamma_{\mathrm{LC}}^{s}} \overline{\boldsymbol{t}}_{i}(\llbracket \overline{\boldsymbol{u}} \rrbracket) N^{m_{i}^{i+1}} d s \pm \\
& \int_{\Gamma_{\mathrm{LU}}^{s}}\langle\overline{\boldsymbol{\sigma}}\rangle \cdot \overline{\boldsymbol{n}}_{i} N^{m_{i}^{i+1}} d s+ \\
& \frac{1}{2} \int_{\Gamma_{\mathrm{LU}}^{s}} \nabla N^{m_{i}^{i+1}} \cdot\left[\left(\llbracket \overline{\boldsymbol{u}} \rrbracket \otimes \overline{\boldsymbol{n}}_{i}\right): \overline{\mathbb{C}}_{0}{ }_{i}^{i+1}\right] d s \pm \\
& \int_{\Gamma_{\mathrm{LU}}^{s}}\left[\left\langle\frac{\beta_{s}}{h_{s}} \overline{\mathbb{C}}_{0}\right\rangle: \llbracket \overline{\boldsymbol{u}} \rrbracket \otimes \overline{\boldsymbol{n}}_{i}\right] \cdot \overline{\boldsymbol{n}}_{i} N^{m_{i}^{i+1}} d s, \\
& f_{\tilde{p} \text { int }}^{e}=\int_{\Omega^{e}}\left[\tilde{p} N^{m}+\nabla N^{m} \cdot\left(\boldsymbol{c}_{\mathrm{g}} \cdot \nabla \tilde{p}\right)\right] d V \\
& \text { and } f_{p \text { int }}^{e}{ }^{m}=\int_{\Omega^{e}} p N^{m} d V .
\end{aligned}
$$

The interface forces $\boldsymbol{f}_{\overline{\mathbf{u}} \mathrm{L}}^{s} m_{i}^{i+1}$ (B.3) arising from the DG/ECL formulation are evaluated for the degrees of freedom belonging for both the plies $i$ and $i+1$. In their expression, " \pm " holds for "+" on the " $i+1$ "-side and for "--" on the " $i "$-side. This is a particularity of the presented implementation, which duplicates the degrees of freedom at a common node instead of duplicating the nodes at common interfaces as this is usually done for cohesive methods. In their integration the shape functions $N^{m_{i}^{i+1}}$ are volume shape functions evaluated at the integration points of the interface elements. Due to the symmetrization terms all the nodes of the 2 neighboring tetrahedra have force contributions, and not only the nodes of the common interface. For quadratic tetrahedra, the evaluation of the interface forces requires a full 6 -point integration at the interface element in order to avoid spurious penetration modes as shown in [73].

\section{Appendix C. Linearization of the non-local finite element forces}

In the case of a staggered resolution, only the non-local contribution of the system of Eqs. (24-25) required to be linearized for the Newton-Raphson resolution. At iteration $i$, this reduced system linearized around the non-local field $\tilde{\overline{\mathbf{p}}}^{i}$ reads

$$
\left[\mathbf{K}_{\tilde{p} \tilde{p}}-\mathbf{K}_{p \tilde{p}}\right][\delta \tilde{\overline{\mathbf{p}}}]=\left[\mathbf{f}_{p \operatorname{int}}\left(\tilde{\overline{\mathbf{p}}}^{i}\right)-\mathbf{f}_{\tilde{p} \text { int }}\left(\tilde{\overline{\mathbf{p}}}^{i}\right)\right] .
$$


The linearization of the elementary forces (B.4-B.5) can be obtained as

$$
\begin{array}{rlr}
\boldsymbol{K}_{\tilde{p} \tilde{p}}^{e} \operatorname{int}^{m n} & =\frac{\partial f_{\tilde{p} \text { int }}^{e} \tilde{p}^{m}}{\partial \tilde{p}^{n}} \\
& =\int_{\Omega^{e}}\left(N^{m} N^{n}+\nabla N^{m} \cdot \boldsymbol{c}_{\mathrm{g}} \cdot \boldsymbol{\nabla} N^{n}\right) d V, \\
\boldsymbol{K}_{p \tilde{p} \text { int }}^{e}{ }^{m n} & =\frac{\partial f_{p \text { int }}^{e}{ }^{m}}{\partial \tilde{p}^{n}}=\int_{\Omega^{e}} \bar{C}_{p} N^{n} N^{m} d V, \quad(\mathrm{C} .2)
\end{array}
$$

where the material tensor $\bar{C}_{p}=\frac{\partial p}{\partial \tilde{p}}$ follows from the MFH scheme and is reported in [28]. 